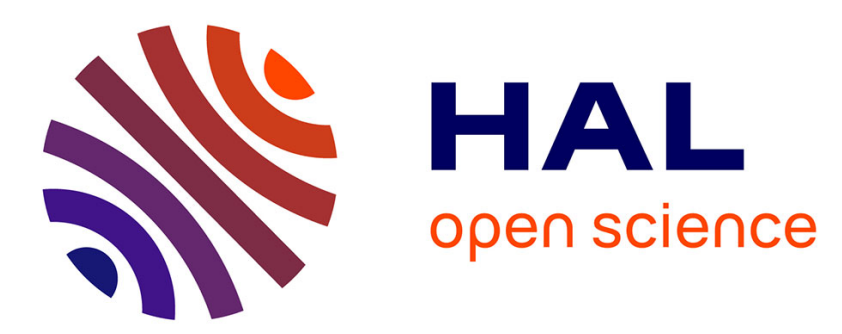

\title{
Application of the infrared extinction to a swirled air/ethanol spray downstream from a turbojet injection system
}

Virginel Bodoc, Olivier Rouzaud

\section{- To cite this version:}

Virginel Bodoc, Olivier Rouzaud. Application of the infrared extinction to a swirled air/ethanol spray downstream from a turbojet injection system. Atomization and Sprays, 2018, 28 (3), pp.255-279. 10.1615/AtomizSpr.2018021180 . hal-01874292

\section{HAL Id: hal-01874292 https://hal.science/hal-01874292}

Submitted on 14 Sep 2018

HAL is a multi-disciplinary open access archive for the deposit and dissemination of scientific research documents, whether they are published or not. The documents may come from teaching and research institutions in France or abroad, or from public or private research centers.
L'archive ouverte pluridisciplinaire HAL, est destinée au dépôt et à la diffusion de documents scientifiques de niveau recherche, publiés ou non, émanant des établissements d'enseignement et de recherche français ou étrangers, des laboratoires publics ou privés. 


\title{
Application of the Infrared Extinction to a Swirled Air/Ethanol Spray Downstream from a Turbojet Injection System.
}

\author{
Virginel Bodoc ${ }^{\mathrm{a}}$, Olivier Rouzaud ${ }^{\mathrm{a}}$ \\ ${ }^{a}$ ONERA - The French Aerospace Lab F-31055, Toulouse, France
}

\begin{abstract}
An investigation of the vapor phase concentration is performed using the Infrared Extinction (IRE) technique on an ethanol spray injected into a heated environment. Experiments are carried out on a confined geometry behind a real- scale injection system. It is composed of a pressure atomizer and an air swirler. The first part of the paper describes the fundamentals of the measurement technique and the experimental procedure. To obtain spatially resolved results, a deconvolution procedure based on Abels algorithm and Tikhonovs regularization is developed. In the second part, the steady measurements performed downstream from the injector provide the radial evolution of the integral vapor molar concentration for various different air temperatures. In addition, a spectral analysis of time- resolved recordings shows that the liquid droplets are moving with the frequency of the Precessing Vortex Core (PVC). In the last part of the article, the local measured values of concentration are compared with the numerical ones. Firstly, the numerical approach is validated for the pure gaseous and two-phase flow behavior. Secondly, the experimental and numerical vapor molar concentrations are presented and discussed.
\end{abstract}

Key words: Makila injector, Infrared Extinction, Abel deconvolution, Tikhonov regularization, vapor concentration, numerical simulation

\section{Introduction}

Fuel evaporation is encountered in a large number of applications, such as internal combustion engines, gas turbines, rocket engines or industrial burners. For all of these applications, the liquid fuel is injected into the combustor and atomized. Due to the evaporation phenomenon, the gaseous mixture is burnt. Generally, the evaporation has a characteristic time greater than that of the combustion; therefore the

Email address: Virginel.Bodoc@onera.fr ( Virginel Bodoc). 
combustion dynamics are controlled by the evaporation rate. Consequently, it is of great importance to have experimental tools that accurately measure the droplet evaporation. In addition, due to the expensiveness of experiments, the aircraft engine industry relies more and more on the use of numerical simulation, for which the physical models need to be validated. Although droplet characteristics (size, velocity and temperature) can be relatively easily measured experimentally by Phase Doppler Anemometry, Rainbow Refractometry and/or Laser Induced Fluorescence, vapor distribution characterization is more challenging.

Considering an evaporating spray under reactive or non-reactive conditions, the vapor concentration is mainly measured by Laser Induced Fluorescence [1], [2]. Nevertheless, the use of this technique implies using complex and expensive high technology equipment, such as tunable lasers and intensified cameras. Although the ability of the technique has been demonstrated for a wide range of applications, its relatively low recording rate ( $\leq 30 \mathrm{~Hz}$ for most commercially available systems) makes it rather suitable for steady flow. In addition, due to its sensitivity to concentration gradients, LIF application for two-phase flows is limited. Given that the measurement principle is based on the fluorescence properties of molecules, the LIF method is also only applicable to a few chemical species. A possible solution to overcome these limitations is based on the use of the vapor absorption properties. In this context, the Infrared Extinction (IRE) technique was developed as a non-intrusive method. It is based on the measurement of the residual laser beam intensities after having crossed a flow. For axisymmetric configurations, the vapor concentration distribution can be inferred from line-of-sight measurements coupled with a deconvolution technique. Since its first application in the 80s, the IRE technique has been applied to many different configurations, including a quite limited number of two-phase flows. The results published in [3], [4], [5], $[6],[7],[8]$ were obtained for various spray configurations and a limited number of vapor concentrations, particle mass loadings and particle sizes. The purpose of this work is to extend the application of the IRE to a real-scale aeronautical spray undergoing evaporation. The size and velocity of the droplets are measured by Phase Doppler Anemometry (PDA), while the gaseous flow has been previously analyzed by Laser Doppler Anemometry (LDA) [9]. The IRE measurements presented here enable the experimental database available for this injection system to be supplemented.

In the second part of the paper, numerical simulation has been performed to assess the experimental data. The simulation has been carried out with the industrial solver CEDRE [10], using a Large Eddy Simulation (LES) approach. The evaporation model implemented in the industrial solver CEDRE is applied, in order to estimate the vapor molar concentration in the test channel. The experimental and numerical values are presented and discussed thereafter.

\subsection{State of the art of the Infrared Extinction technique}

This section provides an overview of the infrared absorption/extinction technique applied on gas/particle flows. To the best of our knowledge, Skinner [11] used infrared detectors for the first time in 1979 to measure the concentration of $\mathrm{CO}$ in moist and dusty atmospheres within coal-fired boiler plants.

A.R. Chraplyvy [12] extended the application of the method based on the absorption/extinction of light for sprays. The objective was to determine the local, time-averaged vapor concentration of an n-heptane spray, despite the influence of droplet scattering. Two He-Ne lasers with wavelengths of respectively 632.8 and $3390 \mathrm{~nm}$ were chosen. The laser beams were overlapped to allow simultaneous attenuation and scattering measurements. A deconvolution method based on the Onion Peeling algorithm was used to extract local values of the vapor concentration from line-of-sight measurements. The use of two laser beams together with a theoretical model enabled the attenuation of the vapor phase to be distinguished 
from that of the dispersed one.

Winklhofer et al. [13] used an experimental set-up similar to that of Chraplyvy to characterize the fuel/air mixture inside combustion chambers under reactive conditions. Flame light contribution was suppressed using spatial and spectral filters. The tested fuels were ethanol and a gasoil blend. Experimental measurements provided extinction levels for both visible and infrared radiations. Liquid and vapor distributions inside the test chamber were deduced from these data. However, quantitative values could not be obtained, due to the lack of additional measurements for the dispersed phase (droplet size and number density).

Significant improvements of the IRE were made by two research teams in Japan and in the USA between 1990 and 1995. In 1991, Adachi et al. [3] proposed using a broadband light source instead of monochromatic laser beams. Narrow wavelengths are separated by band-pass filters. Using three filters, one at the main absorption wavelength $(3.5 \mu \mathrm{m})$ and two others at non-absorbing wavelengths $(3$ and $4 \mu \mathrm{m}$ respectively), it was possible to distinguish between vapor and liquid attenuations. Hence, the scattering amount of droplets was obtained from extinction measurements at the two non-absorbing wavelengths. The mean value was afterwards considered for the calculation of the transmittance at $3.5 \mu \mathrm{m}$.

Drallmeier et al. performed extensive research on IRE [4], [5], [6], [14]. Experiments similar to those of Chraplyvy were performed on an evaporating spray of iso-octane. Droplet distribution, optical thickness, local vapor concentration and vapor mass flux were determined combining infrared attenuation measurements with PDA analysis.

In 1994, Drallmeier presented a simplification of the measurement principle that avoids the calculation of the Mie scattering for each measurement point. This simplification was made possible due to the extinction caused by the dispersed phase for two different wavelengths, 632.8 and $3390 \mathrm{~nm}$ is almost identical for droplets with a diameter greater than $20 \mu \mathrm{m}$. In addition, since the visible radiation is not absorbed by hydrocarbon vapors, the contribution of the dispersed phase to the attenuation of the infrared laser beam can be estimated. The Mie scattering simulations show that the complex refractive index has little impact on the optical thickness ratio under these conditions.

After 1995, IRE has been applied to different multiphase flows, such as fuel sprays in engines [15], [16] and detection of aerosols [17].

Recently, the measurement technique as it was developed by Drallmeier was applied for an ultrasonic spray of n-octane by [8] and the vapor concentration was compared with the values resulting from a numerical simulation.

\section{Experimental approach}

\subsection{Measurement principle of the IRE technique}

The extinction (absorption and scattering) of a laser beam intensity after crossing a medium with randomly distributed particles is theoretically described by the Bouguer-Lambert-Beer (BLB) law, which was derived from laser beam energy conservation [18]:

$$
\frac{I}{I_{0}}=\exp (-\tau)=\exp \left[-\bar{C}_{n} \cdot L \cdot \frac{\pi}{4} \cdot \int_{0}^{\infty} Q_{e x t}(\lambda, n, D) \cdot D^{2} \cdot N(D) d D\right]
$$

where the extinction coefficient $Q_{e x t}$ is written as:

$$
Q_{\text {ext }}(\lambda, n, D)=Q_{\text {scat }}(\lambda, n, D)+Q_{a b s}(\lambda, n, D)
$$

The term $\tau$ represents the optical thickness of the medium. The coefficients $Q_{s c a}$ and $Q_{a b s}$ are the scattering and absorption efficiency factors depending on the light wavelength $\lambda$, the size of particles $D$, 
and the complex refractive index $n \cdot \bar{C}_{n}$ and $N(D)$ are the particle mean density number and the diameter distribution, respectively.

If the continuous phase is absorbing, the extinction effect due to the gas has to be taken into account and the previous equation becomes:

$$
\frac{I}{I_{0}}=\exp (-\tau) \cdot \exp [-\alpha \cdot \bar{c} \cdot L]
$$

where $\bar{c}$ represents the mean molar concentration and $\alpha$ is the molar absorption coefficient of the medium.

The Infrared Extinction (IRE) technique is based on the simultaneous measurement of the laser beam extinction for two wavelengths, $\lambda_{1}$ and $\lambda_{2}$. The value of the first wavelength $\lambda_{1}$ is selected in order to allow an important absorption in the continuous phase. Usually, hydrocarbons display such a behavior for infrared radiation. On the contrary, the second wavelength $\lambda_{2}$ is chosen to be such that the extinction is mostly due to the particle cloud, while the absorption of the continuous phase is quite small. For practical reasons (availability of the laser sources and important absorption coefficients), most of the optical arrangements were developed for $\lambda_{1}=3.39 \mu \mathrm{m}$ and $\lambda_{2}=0.632 \mu \mathrm{m}$. From the measured extinctions, the mean molar concentration is obtained from equations 1 and 3 :

$$
\begin{gathered}
\bar{c}=\frac{1}{\alpha \cdot L}\left[-\tau_{1}-\ln \left(\frac{I}{I_{0}}\right)_{\lambda_{1}}\right]=\frac{1}{\alpha \cdot L}\left[\ln \left(\frac{I}{I_{0}}\right)_{\lambda_{2}} \frac{\tau_{1}}{\tau_{2}}-\ln \left(\frac{I}{I_{0}}\right)_{\lambda_{1}}\right] \\
\bar{c}=\frac{1}{\alpha \cdot L}\left[\ln \left(\frac{I}{I_{0}}\right)_{\lambda_{2}} \frac{Q_{e x t}\left(\lambda_{1}, n, D\right)}{Q_{e x t}\left(\lambda_{2}, n, D\right)}-\ln \left(\frac{I}{I_{0}}\right)_{\lambda_{1}}\right]=\frac{1}{\alpha \cdot L}\left[\ln \left(\frac{I}{I_{0}}\right)_{\lambda_{2}} \cdot R-\ln \left(\frac{I}{I_{0}}\right)_{\lambda_{1}}\right]
\end{gathered}
$$

This equation implies that the extinction efficiency ratio $R=\frac{Q_{e x t}\left(\lambda_{1}, n, D\right)}{Q_{e x t}\left(\lambda_{2}, n, D\right)}$ is known beforehand. Different investigations based on the simulation of light diffusion with Mies theory [5] have shown that the extinction efficiency ratio can be assumed to be equal to 1 , independently of the refractive indices of the droplets, if the surface area mean diameter D20 is greater than 20 micrometers. Hence, the IRE technique is reduced to the simultaneous measurement of the laser beam extinction for two different wavelengths.

In practical applications, there are some difficulties in the application of Equation 5. Hence, following van Hulst [18], a detector measuring the laser beam intensity after crossing a gas/particle flow, simultaneously perceives the attenuated energy of the source and scattered light in its field of view (FOV). This effect is more significant for large spheres, because the scattered light is more concentrated near the forward direction.

The limits of the BLB law were experimentally tested by Swanson et al. [19] using a $514.5 \mathrm{~nm}$ laser. The authors proved that the effect of the light scattered by the particles cannot be neglected relative to the collected radiant intensity. This effect is more obvious for high values of the optical thickness and particle size. Nevertheless, by decreasing the FOV of the detector, the amount of the scattered light reaching the sensor may be limited. Under these conditions, the BLB law was proved to be valid for dense media $(\tau>10)$ if the FOV half-angle satisfies the so-called Hodkinson criterion: $\theta_{1 / 2}<7^{0} \frac{\lambda}{D}$ where $\lambda$ corresponds to the wavelength of the radiation and $\mathrm{D}$ to the diameter of droplets. As shown by Gouesbet et al. [20] this condition has been proven for single scattering particles and is expected to remain valid if multiple scattering must be taken into account.

\subsection{Experimental setup}

The vapor concentration was measured with IRE techniques, while the droplet size and velocity measurements were performed by PDA. 
Figure 1 presents a view of the experimental set-up. It consists in a plenum chamber, containing an injection system provided by TURBOMECA, a test chamber and an exhaust channel. The chamber has a square cross-section of $129 \mathrm{~mm}$ x129 mm. The side walls of the test chamber are optical windows, transparent for visible and IR radiation (GE 214 fused quartz). Gas is injected through a radial swirler while a pressure-swirl fuel injector enables liquid to be injected into the test chamber. The nozzle tip of the simplex injector is located at the entrance of the test chamber. The injection system is placed on the left side of the image and the main flow is from left to right.

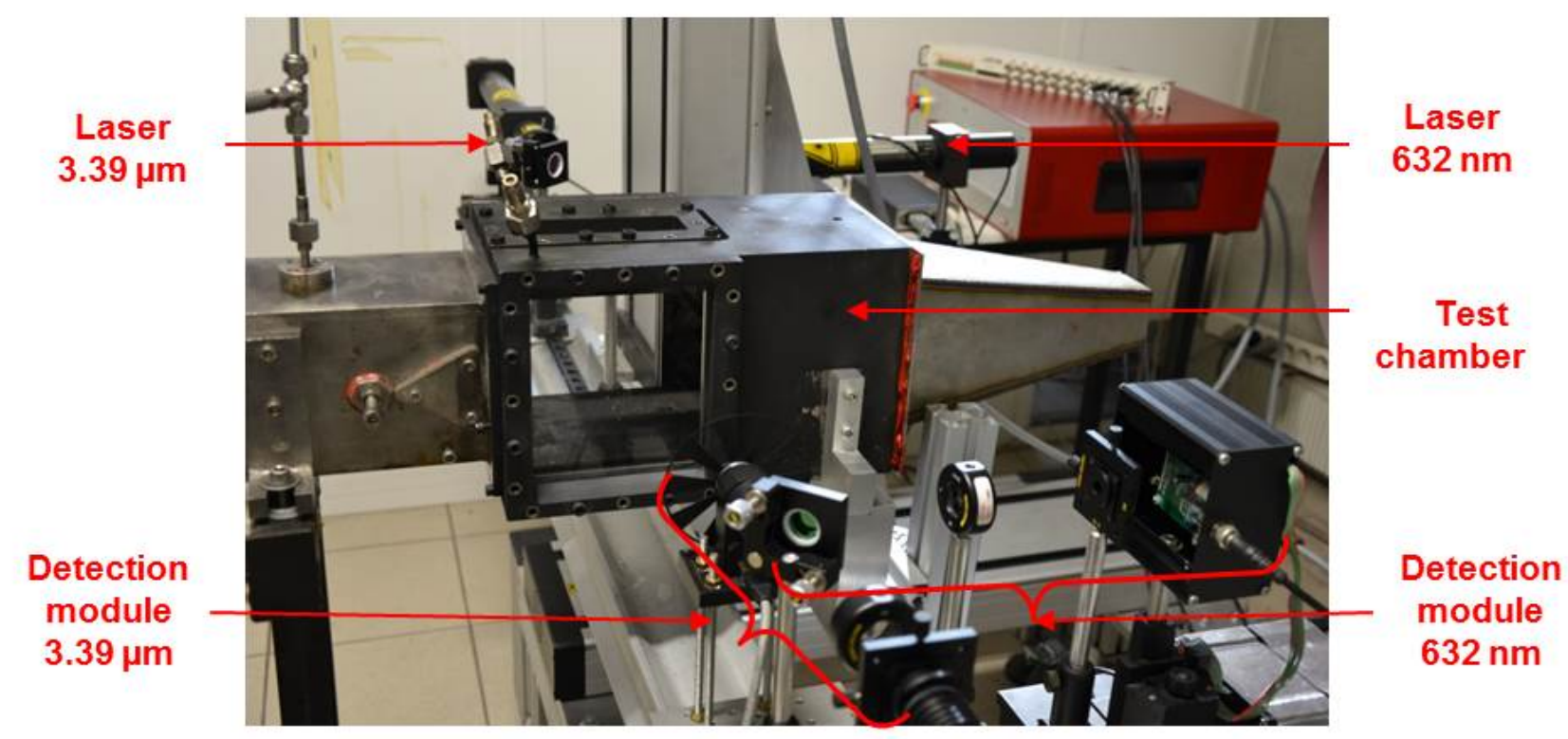

Figure 1. Experimental setup

Concerning the operating conditions, all tests have been done at atmospheric pressure with three different gas temperatures $130{ }^{\circ} \mathrm{C}, 150^{\circ} \mathrm{C}$ and $170^{\circ} \mathrm{C}$. These temperature values prevent the formation of a liquid film on the optical windows and provide some insight into the influence of the temperature on liquid vaporization. The air mass flow rate is equal to $105 \mathrm{Nm} 3 / \mathrm{h}$ (normal cubic meter per hour) . Such a choice is consistent with previous measurements [9] and it enables the existing experimental database to be completed. The liquid fuel mass flow rate is equal to $1.2 \mathrm{~g} / \mathrm{s}$. For the sake of simplicity, ethanol is used as fuel. The molar absorption coefficient (decade value) of the ethanol vapor was previously measured in [21] at ambient pressure and for different values of temperature. It was found that for temperatures less than $100^{\circ} \mathrm{C}$ the mean value of this coefficient may be considered as $\alpha=3.2 \mathrm{~m}^{2} / \mathrm{mol}$ if an error of about $6 \%$ is assumed.

A Dantec FlowMaster Model two-channel PDA has been used for this study. The measurement of two components of the velocity is performed using two orthogonally oriented laser beams of different wavelengths (532 and $671 \mathrm{~nm}$ ). PDA provides droplet size, droplet velocity and the volume flux by measuring a statistically significant number of particles passing through the probe volume. The receiver (FiberPDA Model) was placed at a 30 degree angle from the emitter optical axis. A number of 20,000 drops has been collected at most of the locations, except in the regions of the spray where the droplet number density is very low, close to the axis center or at radial locations greater than 50 millimeters. The acceptance rates were typically greater than $90 \%$. 


\subsection{IRE optical setup}

The Infrared Extinction optical set-up is presented in figure 2. Two light sources are used: one in the visible spectrum $\left(\lambda_{2}=632 \mathrm{~nm}\right)$ and the second one in the infrared $\left(\lambda_{1}=3.39 \mu \mathrm{m}\right)$. The two laser beams are overlapped through a silica plate beamsplitter T3.39/R.633 from Melles Griot. A pinhole is placed on the optical path before the test chamber, in order to provide the same diameter for both beams. A chopper generates a time dependent signal that is used as a trigger source by the acquisition system. The transmitted laser beams were focused onto photodetectors. The visible radiation detector is a Si-type photodiode, while the amount of infrared radiation is measured by a PbSe photoconductive detector. In order to minimize thermal noise, the IR detector is embedded in a thermoelectric heat sink that regulates the operating temperature. In addition, in order to increase their dynamics, the two detectors are connected to pre-amplifying lock-in circuits. The conditioning and storage of the pre-amplified signals are provided by an acquisition system from National Instruments, which is controlled by a Labview-based application. The IR receiver is protected by an ND filter with an optical density of 1.5 , in order to set the detector at a correct operating point. The visible detector is protected by a filter with an optical density of 1 . Thus, overexposure of the sensitive elements is avoided. In order to perform spatially resolved line-of-sight measurements, a two-axis displacement system drives the motion of the optical set-up. The alignment of the laser beams was performed with the razor blade technique.

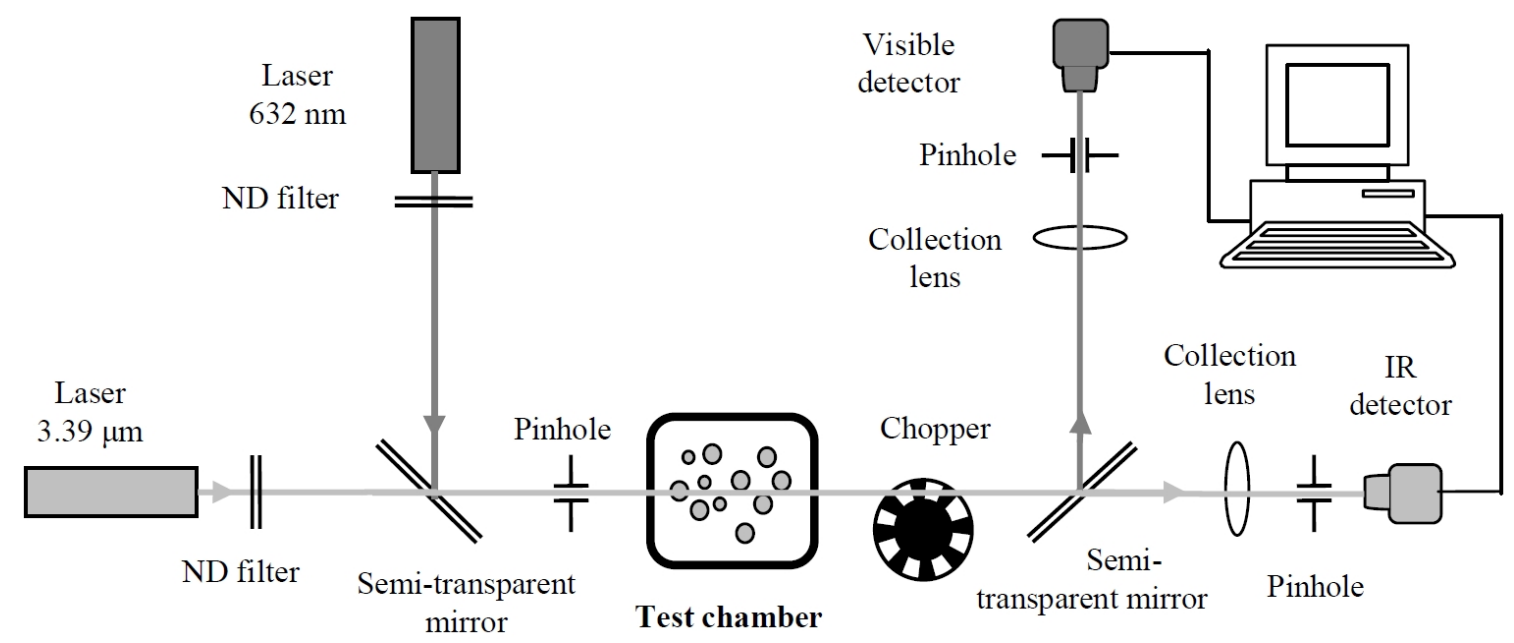

Figure 2. Optical setup

As it has been said previously, it is theoretically possible to express the extinction of light from a point source using the BLB law. Nevertheless, in practice, the detectors measure the attenuated light of the source plus a certain amount of light scattered at small angles. These angles are defined by the FOV of the detector. For the optical setup presented here, the amount of scattered light seen by the detectors has been minimized by placing lens-pinhole assemblies before the detectors. The characteristics of these components are listed in Table 1 .

Using the values from this table, it is of interest to check the applicability of Hodkinsons criterion for this experimental setup. Figure 3 plots the limits of the FOV for the two wavelengths, as defined by the Hodkinson criterion for different sizes of droplets. The same graph presents the FOVs of the optical arrangement as calculated with the elements from table 1. It can be observed that the amount of 
Table 1

Characteristics of the lens-pinhole assemblies

\begin{tabular}{ccc}
\multicolumn{3}{c}{$\lambda_{1}=632 n m \lambda_{2}=3.39 \mu m$} \\
\hline Lens focal length [mm] $f$ & 50 & 100 \\
Pinhole radius [mm] $r$ & 0.4 & 0.15 \\
FOV [degrees] $\theta_{1 / 2}$ & 0.229 & 0.043 \\
\hline
\end{tabular}

scattered light entering in the detector may be considered as negligible for droplets smaller than $100 \mu \mathrm{m}$ in diameter, both for visible and infrared radiation.

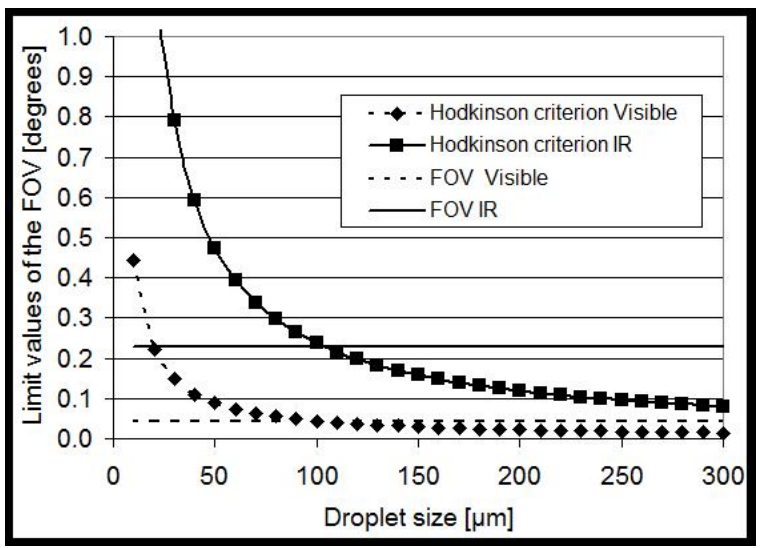

Figure 3. Evolution of the Field-of-View limits with the droplet size for the two wavelengths

Moreover, from the values in the Table 1 , it can be observed that the ratio $\frac{r}{\lambda f}$ is kept constant for both the infrared and visible transmission measurements. From the theory of light diffusion by transparent spheres, the same amount of scattered light will enter the detectors [14], [18] without any influence on the extinction efficiency ratio $\mathrm{R}$. With this optical set-up the reference beams intensities $I_{0}$ and attenuations cannot be measured simultaneously. The solution consists in the measurement of the incident laser beam intensities before any measurement of the attenuation through the medium in the absence of droplets or vapors.

As part of this work, droplet sizes have been measured by PDA and the surface mean droplet diameter $D 20$ has been found to be greater than 20 micrometers. Applying Drallmeier's criterion, the $R$ ratio can be considered to be equal to 1 , which leads to errors as large as $10 \%$ in the measured vapor concentration values. Moreover, droplet sizes range between 0-60 micrometers. According to the results from Figure 3, the multiple scattering effect is neglectible and the Hodkinson's criterion is satisfied.

The measurement grid is represented in Figure 4. The ethanol vapor concentration was measured in various planes from the injector nozzle: 12, 26, 56 and 86 millimeters. In each section, the spatial resolution following the OY axis is 2 millimeters. Due to the axisymmetry of the geometry, only the positive region of the $\mathrm{OY}$ axis was investigated.

\subsection{Description of the reconstruction method}

In line-of-sight (LOS) measurements, vapor concentration is inferred from the extinction of laser beams as they pass through the spray. An inverse problem must be solved to recover local values of the concentra- 


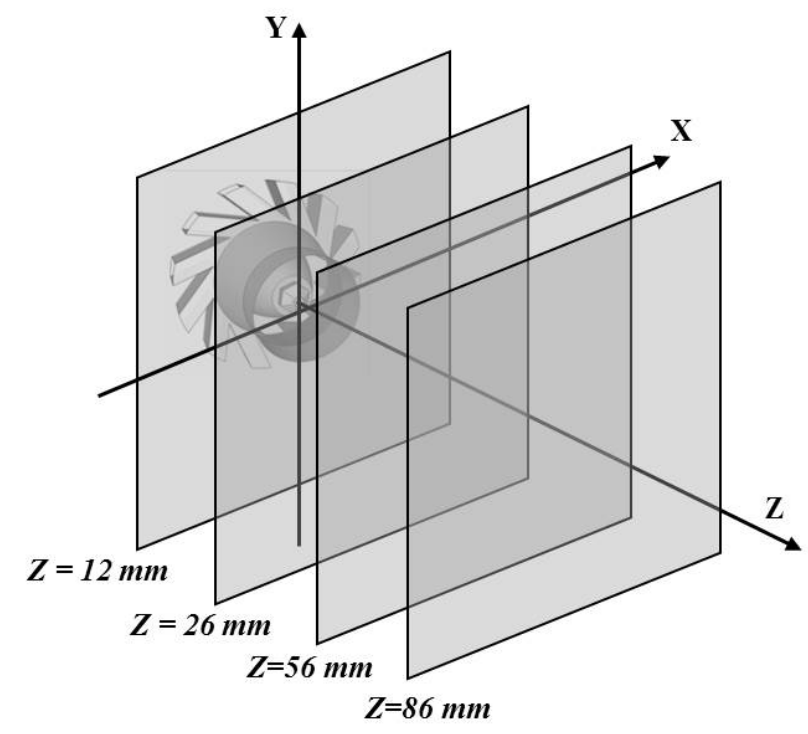

Figure 4. Measurement grid

tion from the optically collected data. Assuming an axisymmetric flow greatly simplifies the reconstruction procedure. For an axisymmetric spray (see the image on the left in Figure 5), the relationship between projection value $C(y)$ and its corresponding radial concentration $c(r)$ is given by Abel's integral equation:

$$
C(y)=2 \int_{y}^{\sqrt{L^{2}+y^{2}}} \frac{c(r) r}{\sqrt{r^{2}-y^{2}}} d r
$$

where $y$ is the coordinate of the projected data, $r$ is the radial coordinate and $L$ is the half-width of the section

In usual applications of LOS measurements, the projected data consist of a discrete set of values, and various discretization techniques that transform Abels equation from the continuous domain into the discrete domain have been developed. The simplest scheme is the onion-peeling one, in which the spray is discretized into a number of annular elements and $c(r)$ is assumed to hold a constant value, as illustrated in the image on the left in Figure 5.

Within the framework of this study a modified onion-peeling scheme was applied to account for the square section of the test chamber (see the image on the right in Figure 5). The spray, which is assumed to be axisymmetric, is divided into a number of concentric rings equal to the number of LOS measurements taken. In our case, the number $N$ is fixed at 30 and has been kept constant in all the measurement planes. The reconstruction begins at the outer edge of the section. The results for the outermost ring are determined from the outermost LOS measurement. The vapor and liquid phase distributions were considered axisymmetric across the section, except at the corners where they are assumed to be uniform. This assumption will be confirmed later in this paper by the results of the numerical simulation.

The integral of Equation 6 is rewritten as a summation on $N$ terms, each of them corresponding to an annular element. Given that the concentration is constant over every annular element, the integral calculation is reduced to the estimation of the geometric term $r / \sqrt{r^{2}-y^{2}}$ over the element. The whole process results in the following equation: 

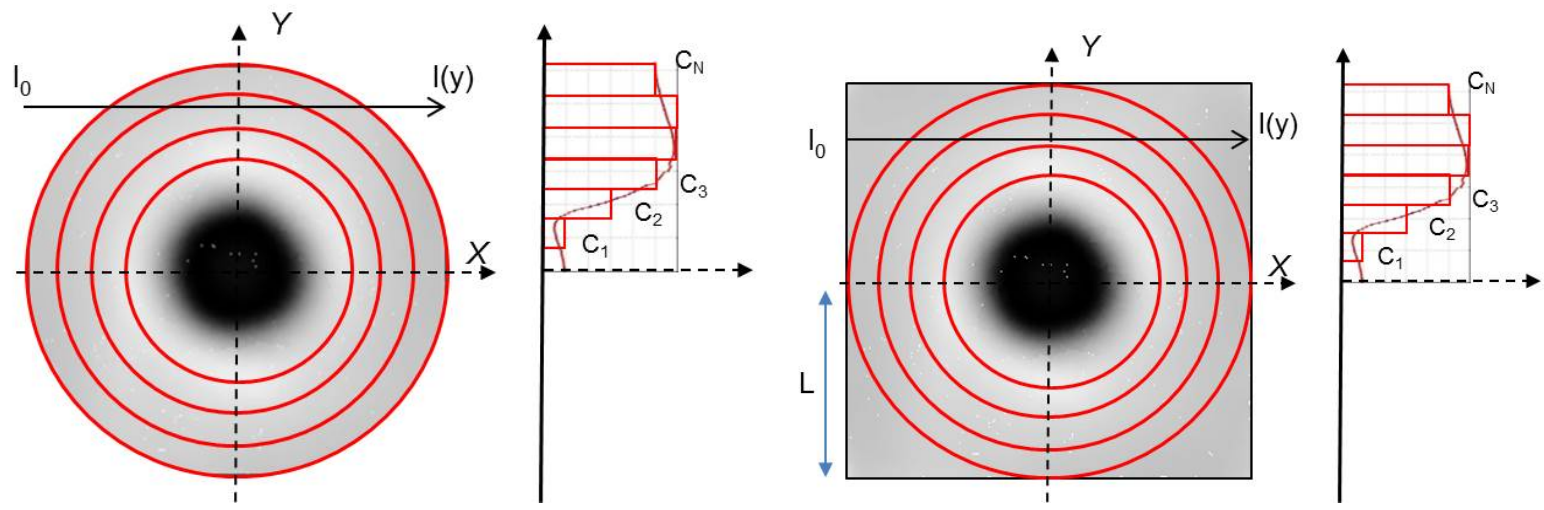

Figure 5. Reconstruction geometry of the axisymmetric spray in a square section tube

$$
\mathbf{A} \cdot \mathbf{x}=\mathbf{b}
$$

where $\mathbf{b}=\left\{C_{1}, C_{2}, C_{3}, \ldots C_{N}\right\}^{T}$ and $\mathbf{x}=\left\{c_{1}, c_{2}, c_{3}, \ldots c_{N}\right\}^{T}$ are discrete sets of, respectively, the projected data and the reconstructed concentrations. These last values are evaluated at the center of the annular elements. The N-matrix A contains geometric terms that are calculated considering the square section of the test chamber, as in the image on the right in Figure 5. The matrix element write as:

$$
A_{i j}= \begin{cases}0, & j<i \\ 2 \Delta r\left[(j-0.5)^{2}-(i-1)^{2}\right]^{1 / 2} & j=i \neq N \\ 2\left\{L-\Delta r\left[(j-1.5)^{2}-(i-1)^{2}\right]^{1 / 2}\right\} & j=i=N \\ 2 \Delta r\left\{\left[(j-0.5)^{2}-(i-1)^{2}\right]^{1 / 2}-\left[(j-1.5)^{2}-(i-1)^{2}\right]^{1 / 2}\right\} & j>i\end{cases}
$$

The resolution of Equation 7 provides the local values of the vapor concentrations. Nevertheless, the direct inversion of the linear system raises numerical issues, because of its stiffness due to the measurement noise. Indeed, small variations in the projected values are amplified by the deconvolution process and may lead to large errors in the reconstructed field. An efficient way of dealing with the ill-posed nature of this problem is Tikhonovs regularization, a process that replaces the original ill-posed problem with an approximate but less ill-posed problem. Various authors [22], [23] have demonstrated that Tikhonov's regularization can stabilize the inversion of Abel's integral equation.

The regularization consists in increasing the size of the linear system (7) with additional rows:

$$
\left[\begin{array}{c}
\mathbf{A} \\
\lambda \cdot \mathbf{L}
\end{array}\right] \cdot \mathbf{x}=\left[\begin{array}{l}
\mathbf{b} \\
0
\end{array}\right]
$$

where:

$$
\mathbf{L}=\left[\begin{array}{ccccc}
1 & -1 & 0 & \cdots & 0 \\
0 & 1 & -1 & & \vdots \\
\vdots & & \ddots & \ddots & 0 \\
0 & \cdots & 0 & 1 & -1
\end{array}\right]
$$


and $\lambda$ represents a regularization parameter that controls the extent of the regularization. Its value has to be chosen as a trade-off between accuracy and stability.

The key to successful regularization lies in choosing a regularization parameter that is well suited to provide a sufficiently smooth and accurate solution of the problem. Hence, $\lambda$ needs to be large enough to stabilize the inversion against perturbation amplification, but not so large as to overwhelm the original equations in $\mathbf{A}$ with the smoothing matrix $\lambda \mathbf{L}$.

Daun [23] proposed a trial-and-error approach for selecting the regularization parameter while Akesson [22] proposed an automatic parameter selection algorithm. Within this work, the second approach has been used.

In this approach, $\lambda$ is chosen using the L-curve, which is formed by plotting the two norms of the solution $\left|L x_{\lambda}\right|$ versus residual vectors $\left|A x_{\lambda}-b\right|$ over the range of possible $\lambda$ values on a log-log scale as shown in Figure 6. Points on the upper-left part of the curve represent under-regularized solutions that accurately solve the linear equation (7) but are also highly oscillatory due to contamination of projected data with measurement errors. Points on the lower right part of the curve belong to over-regularized solutions that are smooth but do not accurately model the concentration profile. A good trade-off is found at the corner of the L-curve.

Figure 6 plots an example of L-curve corresponding to the deconvolution of a synthetic series of projections. The L-curve shown in this figure indicates that the optimum level of regularization corresponds to $\lambda=0.148$. This value was found calculating the point of maximum curvature $k_{\lambda}^{\max }$ for the L-curve as explained in [22]. It must be notted that images from Figure 6 are only presented for demonstration purposes. For the processing of IRE projections, the regularization parameter was evaluated for each measurement plane.
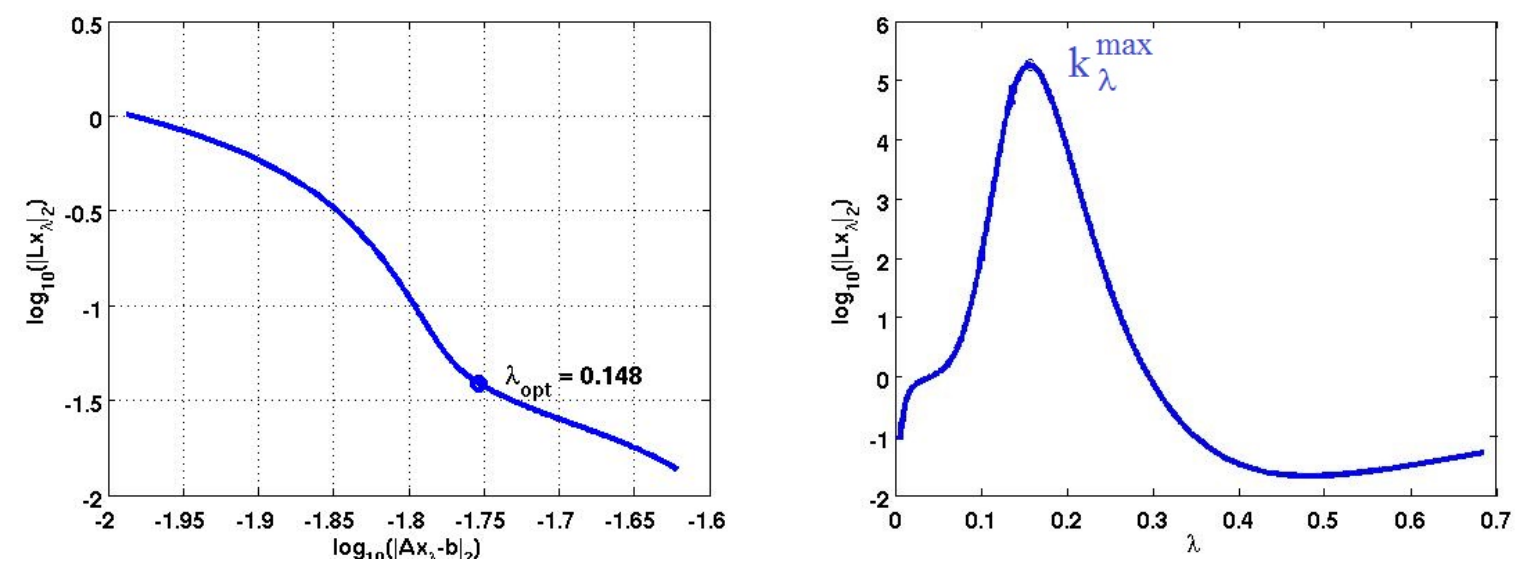

Figure 6. L-curve for $\mathbf{N}=\mathbf{3 0}$ and L-curve curvature

\subsection{Uncertainty analysis and validation of the deconvolution algorithm}

In this paragraph, uncertainty of the IRE results is calculated as a function of the parameters measured in the flow and the deconvolution method. In a first step, the uncertainty analysis will consider only factors involved in achieving LOS measurements. The approach is similar to that of [4]. As indicated by equation 5, the calculation of the projected value of the vapor molar concentration is based on the measurement of four parameters: the molar 
absorption coefficient $\alpha$, the extinction efficiency ratio $R$ and the extinction ratios $I / I_{0}$ for the two wavelengths. Since all the measurements of these parameters are independent, the uncertainty $\Delta \bar{c}$ associated with the vapor concentration $\bar{c}$ is given by the equation:

$$
(\Delta \bar{c})^{2}=\left(\frac{\partial \bar{c}}{\partial \alpha}\right)^{2} \cdot \Delta \alpha^{2}+\left(\frac{\partial \bar{c}}{\partial R}\right)^{2} \cdot \Delta R^{2}+\left(\frac{\partial \bar{c}}{\partial T_{1}}\right)^{2} \cdot \Delta T_{1}^{2}+\left(\frac{\partial \bar{c}}{\partial T_{2}}\right)^{2} \cdot \Delta T_{2}^{2}
$$

where $T_{i}=\ln \left(\frac{I}{I_{0}}\right)_{\lambda_{i}}$ The estimated errors on each of the measured parameters may be expressed as a percentage of the measured value. For example, an error $e_{\alpha}$ on the vapor absorption coefficient can be expressed as:

$$
\Delta \alpha=e_{\alpha} \cdot \alpha
$$

Using this notation and after some mathematical operations, the realtive (normalized) error is:

$$
\left(\frac{\Delta \bar{c}}{\bar{c}}\right)^{2}=1 \cdot e_{\alpha}^{2}+\left(\frac{T_{2} \cdot R}{T_{2} \cdot R-T_{1}}\right)^{2} e_{R}^{2}+\left(\frac{T_{2} \cdot R}{T_{2} \cdot R-T_{1}}\right)^{2} e_{T_{2}}^{2}+\left(\frac{T_{1}}{T_{2} \cdot R-T_{1}}\right)^{2} e_{T_{1}}^{2}
$$

In this equation it may be seen that the sensitivities of the extinction efficiency ratio and the the extinction ratio for the visible radiation are identical.

Using the equation 13 and the experimental extinctions, a detailed error analysis has been performed at different distances from the nozzle. The error sensitivities to the measured parameters at $Z=26 \mathrm{~mm}$ are shown in Figure 7 as a function of the distance to the axis. The sensitivity of the log of the visible extinction is less than one over the entire section. This is due to the fact that the log of extinction for the visible radiation is always inferior to that corresponding to the infrared radiation $\left(T_{2}<T_{1}\right)$ because of the absorption of the vapor phase. Moreover, its value decreases with the absorption in the vapor phase. In other words, for higher vapor concentrations the errors in the measurement of the visible extinction play a lower role in the total error estimation. The sensitivity of the log of the infrared extinction is always greater than one, but decreases with the concentration in vapor phase. From these observations it is desirable to have an extinction mainly due to the absorption in the vapor phase in order to reduce the total error.

For the calculation of the error in the vapor concentration measurement estimations of the errors associated with each parameter were made. As it was discussed before (see section 1), the error in the vapor molar absorption coefficient was estimated at $e_{\alpha}=0.04$.

The error on the extinction efficiency ratio $R$ was estimated using a Mie scattering code for drop size distributions measured by PDA. It was found that $R$ may be approximated to 1.1 with an error of $5 \%: e_{R}=0.05$.

Finally, the error in the measuring the extinction ratios for the two wavelengths was considered by taking into account the spray fluctuations and the laser beam intensity drift. The random error associated with spray fluctuations was estimated at $2 \%$ over a number of 10.000 samples. The error in the laser drift is due to the laser beam intensity variation between the time when the reference intensity $I_{0}$ and the extinguished intensity $I$ are measured. Systematic measurements for different experimental conditions have shown that the maximal error on the log of the intensity ratio is $6 \%$ for infrared radiation and $20 \%$ for the visible radiation and they correspond to the region outside the spray. Smaller values of errors corresponds to the region of the spray.

Given the errors of each of the four parameters and their associated sensitivities, the total error on the vapor molar concentration can be estimated and is shown in Figure 8 . 


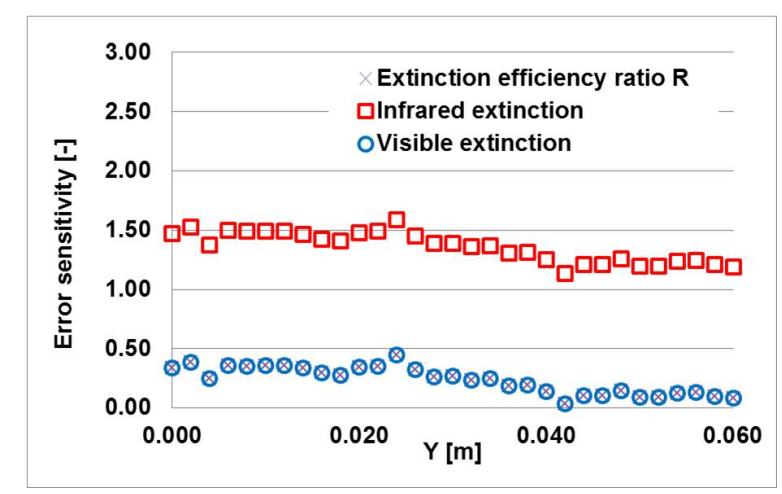

Figure 7. Error sensitivities at $\mathrm{Z}=26 \mathrm{~mm}$.

The total error is less than $14 \%$ over the entire section of the channel. The increase in the central region is due to the increase of the sensitivity of the infrared extinction creating larger errors by Equation 13. Although the calculation has been shown only for $Z=26 \mathrm{~mm}$, similar results were found for the other measurement sections.

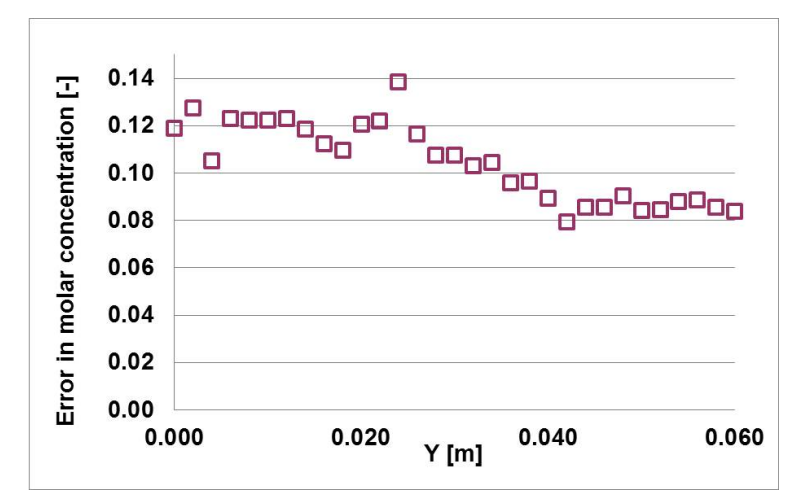

Figure 8. Error in vapor molar concentration at $\mathrm{Z}=26 \mathrm{~mm}$

It must be said that this error analysis concerns only the errors in the line-of-sight vapor molar concentration measurements. The use of the deconvolution algorithm is expected to introduce additional errors which are not necessary independent of the first ones.

In order to estimate the total error, the Tikhonovs regularization method has been applied to invert synthetic data. The field variable is the vapor molar concentration and a radial profile is plotted on the left image in Figure 9 as Original profile. The projected data values are generated by integrating the original profile at discrete radial values (Integral values on the right image). Afterwards, the projected data are contaminated with random noise. The amplitude of the noise corresponds to the error values calculated by Equation 13. An example of such a contaminated data is presented in Figure 9 (right image).

Further, the deconvolution algorithm was run for 1000 series of noise contaminated projections, each time considering optimal values of $\lambda$. The resulting mean and RMS values of local molar concentrations (Deconvoluted profile) are compared with the initial values in the image on the left.

The comparison shows that the reconstruction method overpredicts the concentration in the region close to the axis of the spray, but that the differences are within the uncertainty 
band. Moreover, the reconstruction method seems to amplify the uncertainty from $4-5 \%$ at the border of the spray to $10 \%$ close to the axis.
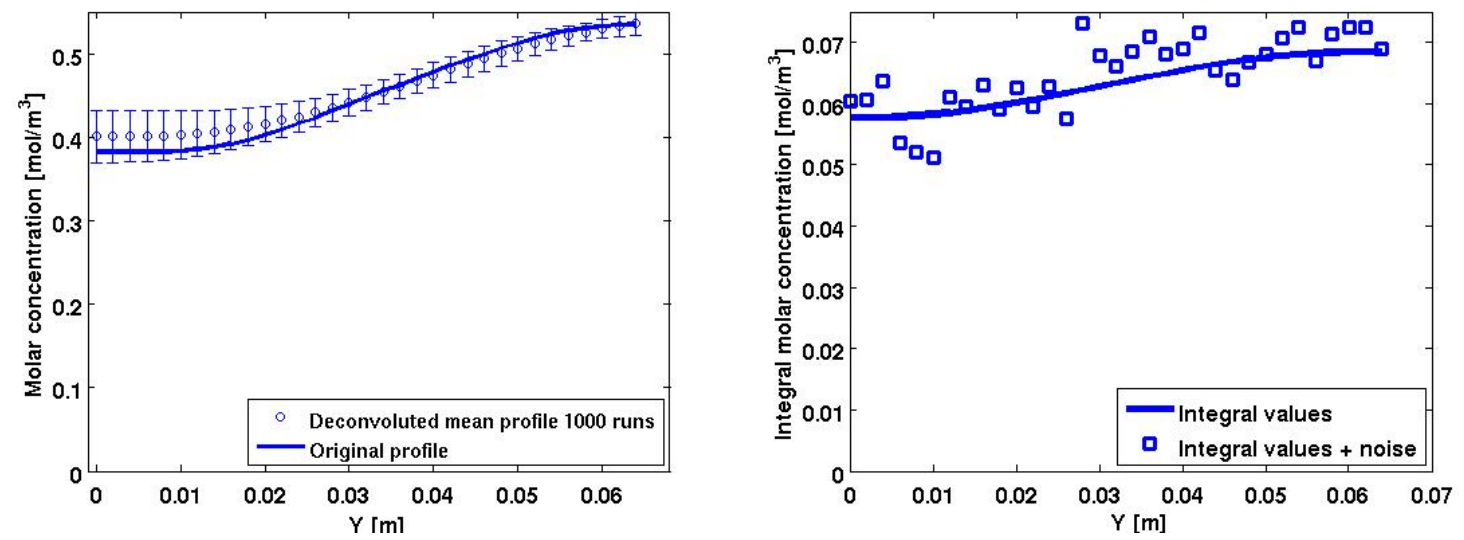

Figure 9. Deconvolution solutions obtained for a perturbed series of integral values. Left image shows radial profiles. Right image shows projected values

\section{Numerical simulation}

\subsection{Description of the computational domain}

The geometry adopted for the numerical simulation corresponds to the experimental configuration (Figure 1). In the left image in Figure 10 a partial view of the computational domain is presented. The plenum chamber where air is introduced is on the left. The liquid injection system and the radial swirler can also be seen inside the plenum chamber. The right part of the image corresponds to the square crosssection test chamber. Air and liquid flows through the circular diffuser that can be seen on the leftmost wall. The main flow goes from left to right.

The right image in Figure 10 shows an overall view of the mesh. The unstructured mesh is composed of 3700000 tetrahedral elements. A preliminary grid study on a different operating condition proved thatthe present mesh is sufficient to reproduce the main features of the gas velocity(mean and r.m.s. values) and to provide a good estimation of the Precessing Vortex Core frequency. The computational grid has been refined in several regions, in order to reproduce the most important features of the flow in terms of turbulence and spray behavior. In particular, from the point of view of the liquid phase analysis, the zone of interest is located in the first part of the square cross-section channel, where liquid atomization, droplet heating, evaporation and dispersion occur. For instance, due to the evaporating properties of the droplets and to the operating conditions (air temperature and the liquid mass flow rate), vaporization is quite significant within the first 100 millimeters of the channel. The Precessing Vortex Core hydrodynamic instability is also located in that region, at the outlet of the diffuser. 

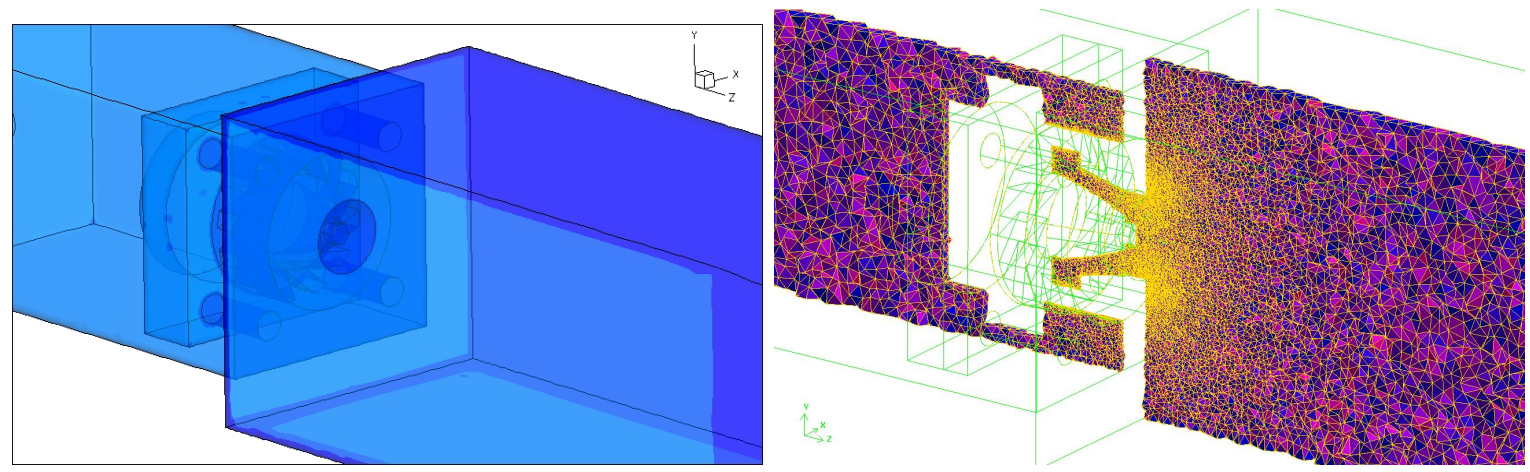

Figure 10. Geometry of the computational domain and global view of the mesh

\subsection{Physical and numerical models}

The numerical simulation has been performed with the in-house industrial software CEDRE [10] developed at ONERA. The software contains different numerical solvers, enabling multiphysics simulations to be performed. In the paper, the Navier-Stokes solver CHARME is used for the gaseous phase and the statistical Lagrangian solver SPARTE [24] is used for the dispersed phase. Since the aim of the simulation was to focus on the vapor concentrations and for the sake of simplicity, the atomization process has not been simulated. Liquid injection will be described later.

The spatial discretization scheme corresponds to a second-order upwind scheme using a Van Leer limiter. A global low-Mach number preconditioning is also applied. Temporal integration is based on an implicit second-order Runge-Kutta scheme. The global time step is equal to $10^{-6}$ seconds. Various authors have shown that swirling jets generate an unsteady and complex flow. The unsteadiness of such a flow has different origins, like the PVC. The generation of vortices, the entrainment of the air from the surrounding medium, and the recirculation are factors that contribute to the complexity of the flow and that justify the use of the LES approach. Since only the large flow structures are solved by LES, the small eddies assumed to be isotropic are modeled by a sub-grid scale (SGS) model. For this simulation, a standard Smagorinsky model is used to account for the unresolved scales of turbulence.

Except at the walls, the boundary conditions for the gaseous phase are of a subsonic inlet/outlet type. At the inlet, the mass flow rate and the total temperature are provided.

In the dispersed phase part, the Lagrangian solver is based on the so-called numerical particle approach, where each numerical particle represents a certain number (weight) oof physical droplets that share the same properties (position, size, velocity and temperature). The motion of each numerical droplet in the gaseous flow field is obtained by solving a system of equations corresponding to the droplet trajectory and the conservation of mass, momentum and energy quantities with a semi-analytical scheme. The time step for the dispersed phase is fixed at $10^{-5}$ seconds. Concerning the momentum equation, the forces acting on the numerical droplet are the drag and the gravitational forces. The Schiller-Neumann model is used for the drag force. The evaporation phenomenon is based on the standard isolated droplet model, where the convection effect is taken into account through the Ranz-Marshall approximation. The infinite conduction model with a Ranz-Marshall correction is used for the droplet heating.

Within the context of this numerical simulation it has been assumed that the droplet dispersion is not influenced by the subgrid turbulence. This assumption is based on the results reported by Fede and Simonin [25]. They showed that the particles dispersion caused by the modeled eddies is very low if the ratio $k_{c} L_{f}>10$, where $k_{c}$ represents the cut-off wave number and $L_{f}$ is the integral length scale. Under these conditions, for a droplet of $10 \mu \mathrm{m}$, which is supposed to be the most sensitive to the subgrid velocity 
fluctuations, the criterion of Fede and Simonin is met.

The droplets crossing the inlet/outlet boundaries are considered to be lost. For the walls, a rebound condition is imposed with a restitution coefficient of 0.8 and rebound angles varying between 3 and $25^{\circ}$.

Finally, mutual influences of the two phases on each other are taken into account through a twoway coupling process. Such a choice is justified by a volume fraction of the dispersed phase of $7 \cdot 10^{-5}$. Coupling between the gaseous and the dispersed phases is achieved by calculating source terms within gaseous equations for the mass, momentum and energy transfer during a time interval or cycle.

\subsection{Modelling of the dispersed phase injection}

In order to model the liquid injection, experimental spray data have been used. The data consists in droplet size and velocity obtained with the PDA system (see Figure 11).

The image in Figure 12 shows an overview of the numerical injector position and of the droplet velocity vectors in the injection plane. The poly-disperse spray is injected into the flow at 12 millimeters from the injector. At that distance, it can be assumed that the primary atomization process is over and that the droplets are formed. Moreover, the spray has an axisymmetric shape, which simplifies the generation of the numerical injector.

A total number of 2405 elementary injectors, distributed in 14 elementary rings, was used for the injection of the dispersed phase. To account for the evolution of the droplet size and velocity with the distance to the axis, for each elementary ring, the size and velocity distributions provided by PDA measurements were used. For simplicity, each distribution is based on 10 classes of sizes.
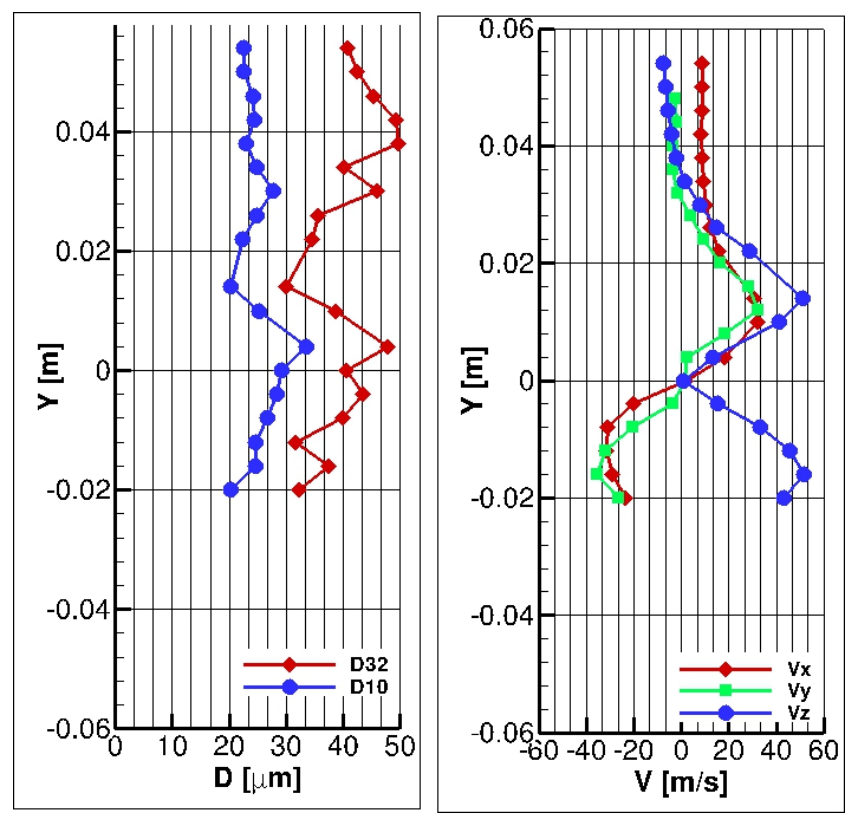

Figure 11. Drop size and velocity components used to model the liquid injection. 


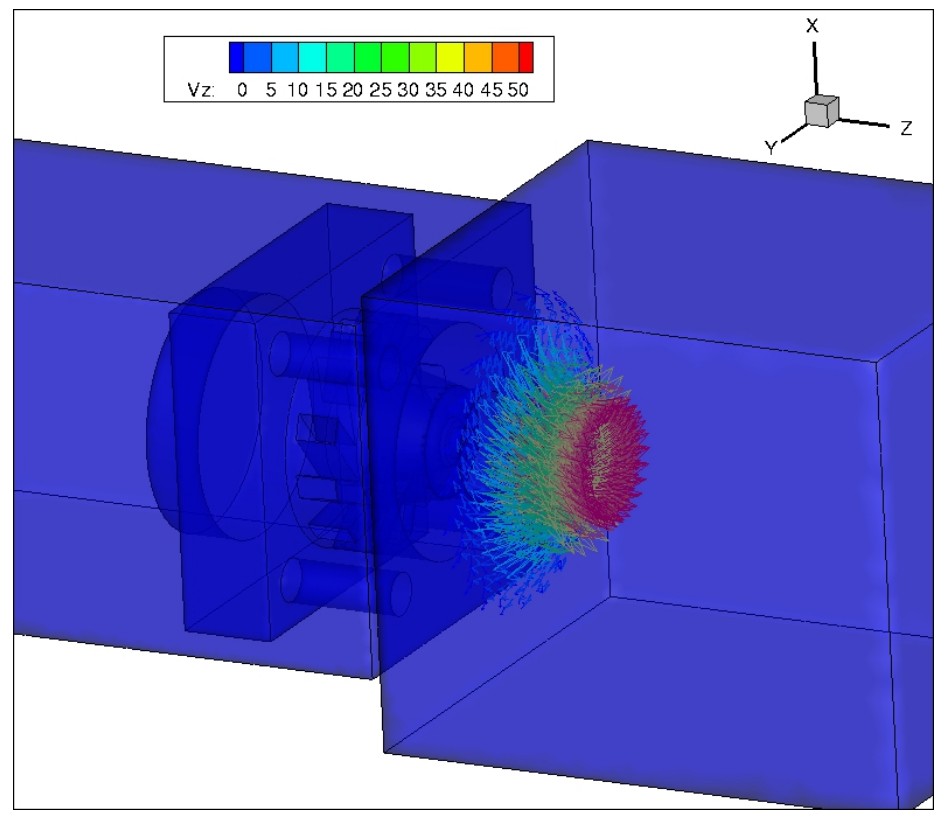

Figure 12. Example of injection points distribution and velocity vectors.

\section{Results}

\subsection{Flow topology}

Figure 13 illustrates the mean flow field obtained with this setup and for the aforementioned operating conditions. A laser tomography technique was used to visualize the ethanol droplets in the median longitudinal section of the chamber by Mie scattering. The droplets were illuminated by a laser light sheet produced by a pulsed laser (Quantel Twins 2x200mJ at $532 \mathrm{~nm}$ ). The images were recorded perpendicularly to the light sheet with a ProX4M camera used in $2 \times 2$ binning mode $(1024 \times 1024$ pixels $)$. It was operated at a frame rate of $5 \mathbf{H z}$ and the integration time was kept at $50 \mu s$. The size of the image was determined in order to have a spatial resolution of 10 pixels/millimeter. The spatial distribution of the volume fraction of droplets was calculated over 500 instantaneous images. The injection system is arranged on the left side of the image and the main flow is from left to right. The swirled flow produces some characteristic structures in the flow, like the central recirculation zone and corner toroidal recirculation zone. The ethanol droplets are located outside of these zones. The highest fraction of the liquid phase is located in the region close to the injector nozzle. Downstream, the liquid fraction decreases due to the vaporization and turbulent dispersion mechanisms.

\subsection{Experimental Results}

The main issue of this paper concerns the measurement of ethanol vapor concentration. The residual intensities provided by the visible detector were processed initially. According to the theory of light scattered by transparent spheres, the light radiation received by the detector is attenuated only by the ethanol droplets and is proportional to their surface. In Figure 14 plots the radial evolution of the 632 nanometer laser beam transmission after crossing the spray at 12 millimeters from the nozzle. A minimal 


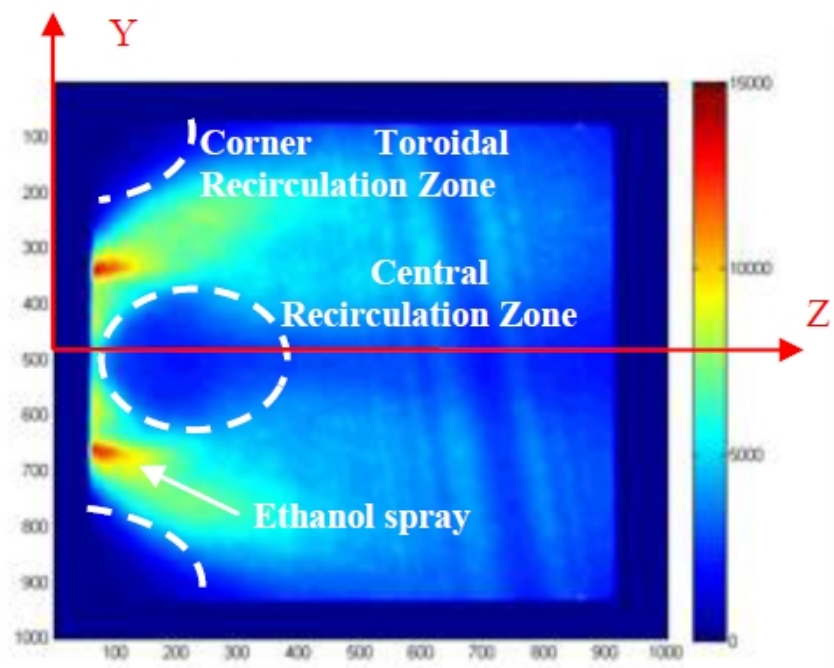

Figure 13. Mean two-phase flow structure. Axis units are expressed in $0.1 \mathrm{~mm}(1000 \mathrm{u} .=100 \mathrm{~mm})$

transmission (or maximal attenuation) is observed at about a radial distance of 20 millimeters from the axis which corresponds to the highest volume fraction of liquid. This observation is consistent with the distribution of scattered light intensity, which is proportional to the droplet surface, as illustrated in Figure 13. Beyond 35 millimeters, the transmission is close to $100 \%$ since only a small amount of droplets is present in this area.

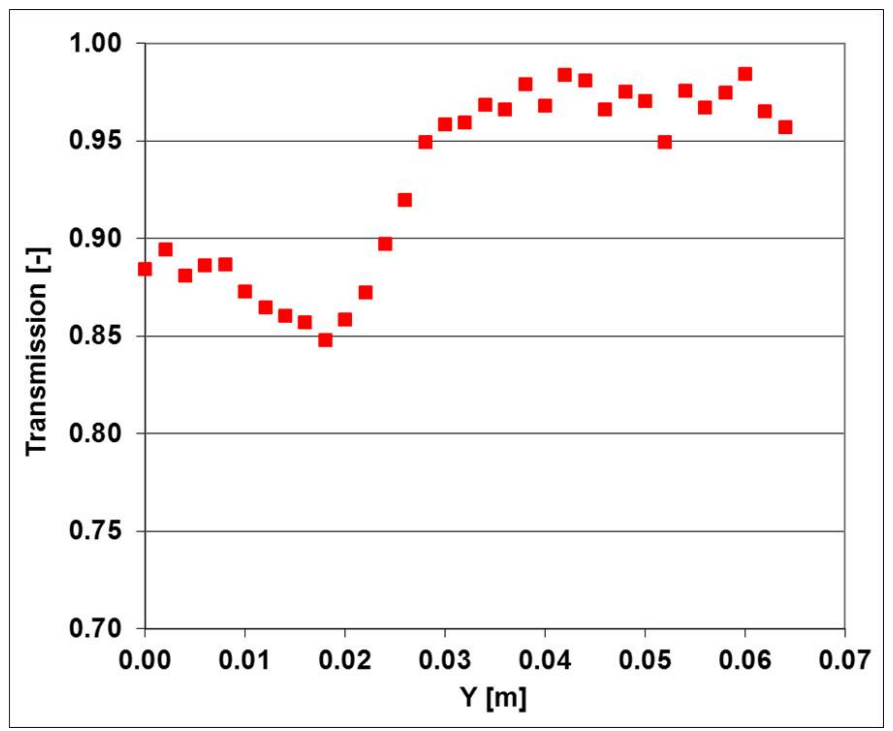

Figure 14. Visible laser beam transmission for various air temperatures at $\mathrm{Z}=12 \mathrm{~mm}$

The IRE system is designed to record data with a sampling rate as high as $10 \mathrm{kHz}$. This allows the unsteady behavior of the ethanol spray downstream from the injector to be analyzed. In order to focus only on the droplet motion, a spectral analysis of the non-chopped signals provided by the visible detector has been performed. FFT is applied to the time-dependent signal, where the mean value has been discarded. 
The power spectral density obtained at a distance of $\mathrm{Z}=12$ millimeters from the nozzle tip, and for a radial position $\mathrm{Y}=20$ millimeters is plotted in Figure 15.

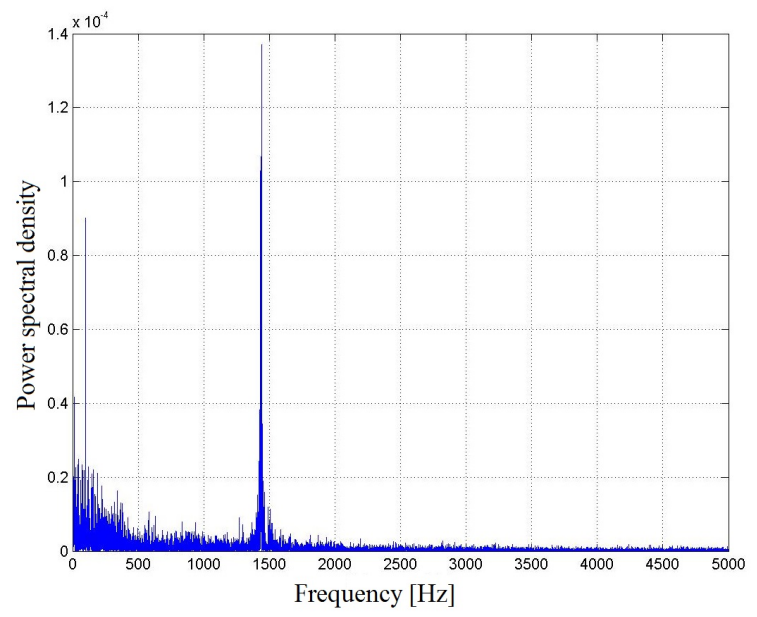

Figure 15. Power spectral density of the visible signal recorded at $\mathrm{Z}=12 \mathrm{~mm}$ and $\mathrm{R}=20 \mathrm{~mm}$.

The graph shows the existence of a sharp frequency peak at 1450 Hertz. Further analysis at different radial positions provides the same frequency. Nevertheless, the peak amplitude varies with the radius reaching a maximal value at $\mathrm{Y}=20$ millimeters. This evolution can be explained by the geometry of the spray with the help of Figure 13. At a radial distance of 20 millimeters from the axis, the optical path crosses the mixture layer of the spray hollow cone, where the amount of liquid fraction has significant variations over time. In order to identify the origin of these oscillations, Figure 16 presents the characteristic frequency evolution with the air mass flow rate that is written as a corrected flow (WR number). The linear evolution of the frequency value with the gas mass flow rate, proves the hydrodynamic origin of the oscillation. Moreover, after comparison with results obtained by time-resolved PDA [26] it was concluded that it corresponds to the Precessing Vortex Core (PVC) instability.

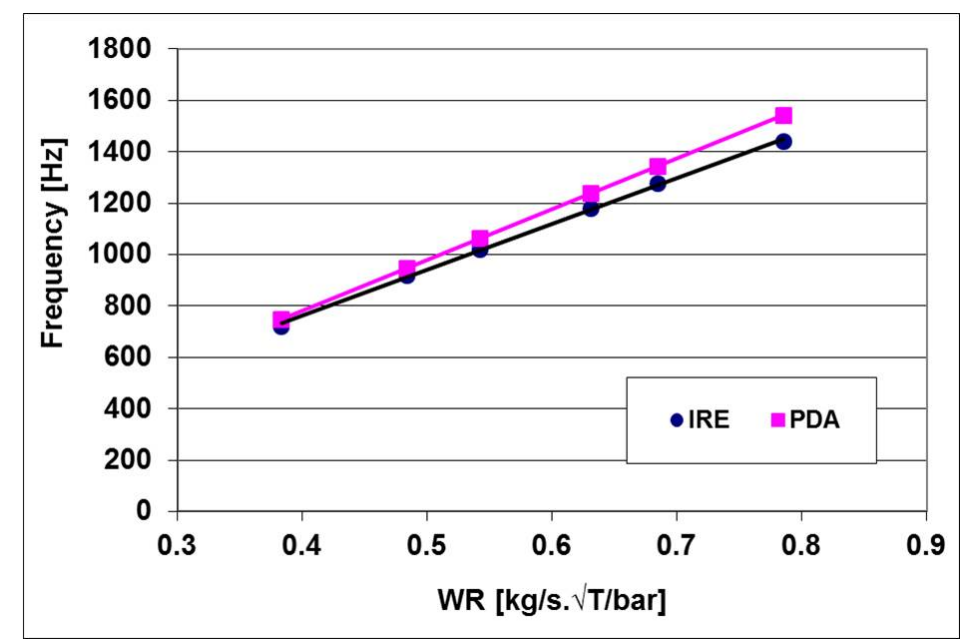

Figure 16. Comparison of PVC frequency measured by IRE and PDA 
Secondly, the residual laser beam intensities for the two wavelengths have been processed, in order to estimate the vapor concentration. The radial distributions of the integrated molar concentration of ethanol vapors at various distances from the injector nozzle are plotted in Figure 17. A large dispersion of the measured values can be observed, which is coherent with the uncertainty analysis done in section 2.5 $(8-12 \%$ of the mean value). For the three measurement planes, the vapor concentration increases with the distance to the axis. This tendency is due to the hollow cone shape of the spray. The various curves show that there is almost no variation in the vapor concentration and, implicitly, there is no influence of the gas temperature on the droplet evaporation rate.
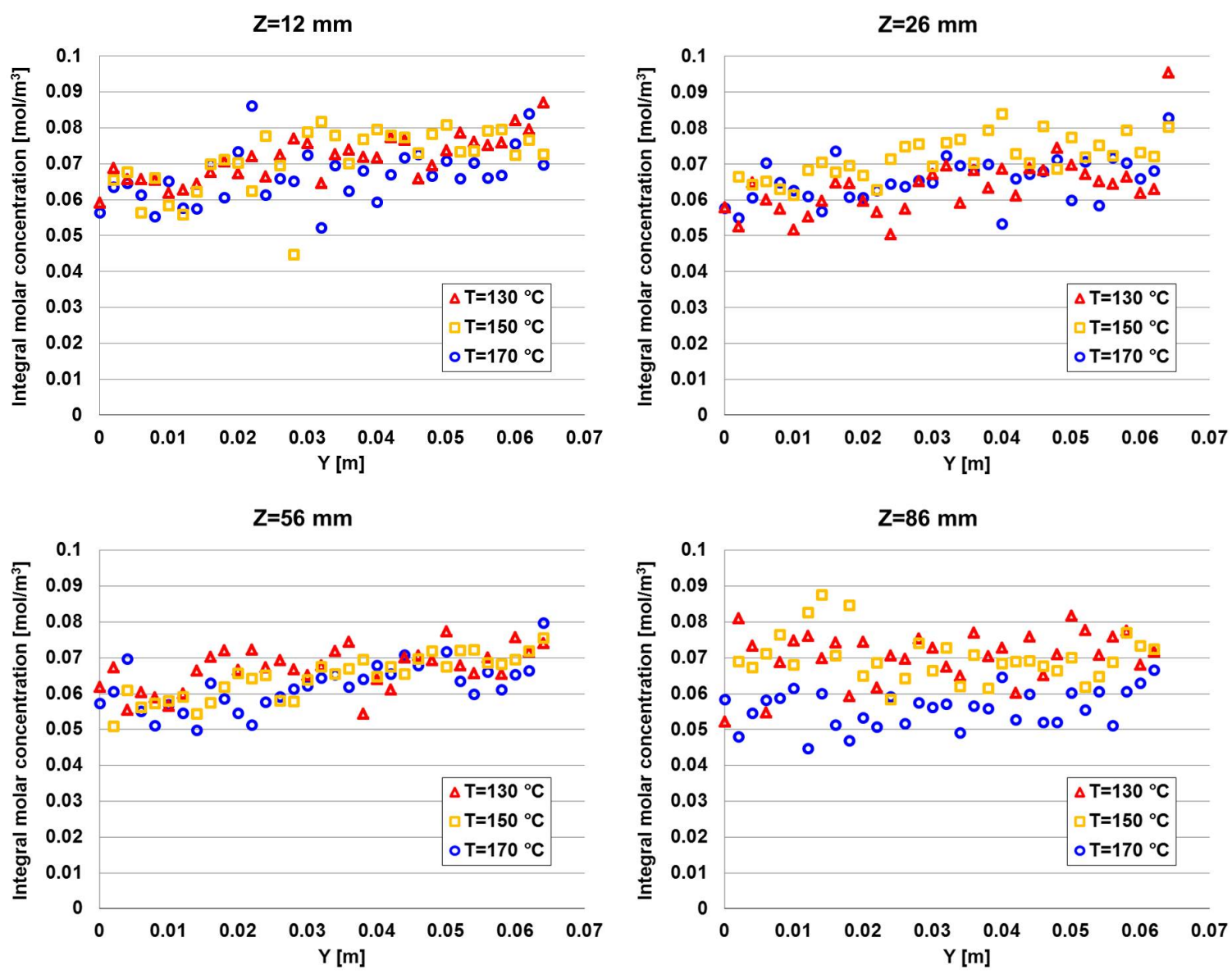

Figure 17. Evolution of the integral molar concentration of ethanol vapors at various distances from the injector outlet.

In order to obtain the local values of the ethanol vapor concentration, the reconstruction method (onionpeeling + Tikhonov regularization) was applied. Given that the results for the three air temperatures are very close, with differences smaller than the method errors, only the values obtained for $T_{a i r}=130{ }^{\circ} \mathrm{C}$ will be presented and compared with the numerical values in Section 4.3.2. 


\subsection{Numerical Results}

\subsubsection{Pure gaseous flow}

The calculation method is based on the experimental process. Initially, the simulation of the gaseous flow is performed during a time period sufficiently large to obtain a fully established gaseous flow. By fully established flow, we mean that the main characteristics of the flow have to be reproduced by the LES calculation. In our case, the physical time of the simulation is equal to 1.1 seconds. It corresponds to 5 residence times, where the residence time is based on the bulk velocity $(\mathrm{m} / \mathrm{s})$ and on a characteristic length equal to $0.3 \mathrm{~m}$. Once the gaseous flow is established, the numerical simulation of the two-phase flow begins.

Assessment of the numerical simulation is based on the comparison with respect to the experimental data of Lecourt [9].The first comparison relates to the averaged values of the axial velocity and its r.m.s. counterpart. The averaging process is performed during a time interval equal to 500 milliseconds. Figure 18 presents the normalized axial velocity versus the transversal coordinate at four different sections, located respectively at $Z=8,26,56$ and 86 millimeters from the chamber end. The normalization velocity is equal to $V_{o}=130 \mathrm{~m} / \mathrm{s}$. Numerical results are in good agreement with the experimental data, capturing the main features of the flow. The first three sections show clearly the presence of the central recirculation zone, as well as the presence of the lateral recirculation zone. In addition, the behavior of the high-sheared region is nicely reproduced. In particular, the numerical results provide a very good prediction of the velocity extrema (in modulus and in position) and a good description of the jet expansion. Due to the section jump, the flow decelerates between $Z=8$ and $Z=86$ millimeters to reach its bulk velocity further downstream. Profiles of the axial r.m.s. velocity are presented in figure 19. Although the numerical results underpredict the maxima in the first section and are slightly overpredicted for the others, comparisons are still very satisfactory. These results demonstrate that the flow is very turbulent due to the high level of the r.m.s values compared to its mean counterpart. Note that the comparisons of the radial and tangential velocities (mean and r.m.s.) provide similar conclusions.

A second point of interest concerns the flow unsteadiness. As it has been previously mentioned, in such a configuration, a Precessing Vortex Core appears at the inlet of the test chamber. Recording the radial velocity fluctuations during the numerical calculations at some discrete points inside the chamber enables the PVC frequency to be found with an FFT. The dominant frequency is close to 1450 Hertz, the experimental value yielded by the analysis of the visible detector signal. All of these comparisons prove that the LES simulation reproduces the main features of the gaseous flow and provides a valuable initial solution for the two-phase flow simulation.

\subsubsection{Two phase flow}

Two-phase flow simulation was performed over 355 milliseconds. Only the last 300 milliseconds have been used to obtain mean values. In each cell of the computational mesh, an exponential moving average accounts for each droplet that crossed the cell and enables spray characteristic values, like the droplet diameter or the droplet velocity, to be defined.

Figure 20 shows a comparison of the droplet longitudinal velocity with respect to the transversal coordinate in four sections, located at 13, 26, 56, and 86 millimeters from the end of the chamber. As for the pure gaseous phase, numerical values provide a good prediction of the velocity extrema position. For the sections at 26 and 56 millimeters the simulation underpredicts the experimental data, although the shapes of the profiles are similar. Comparisons on the radial and tangential velocity components provide the same level of agreement.

The next figure (Figure 21) describes the profiles of the arithmetic diameter D10. The good agreement in the first section is expected since the numerical droplets are injected at the section $Z=12$ millimeters. 

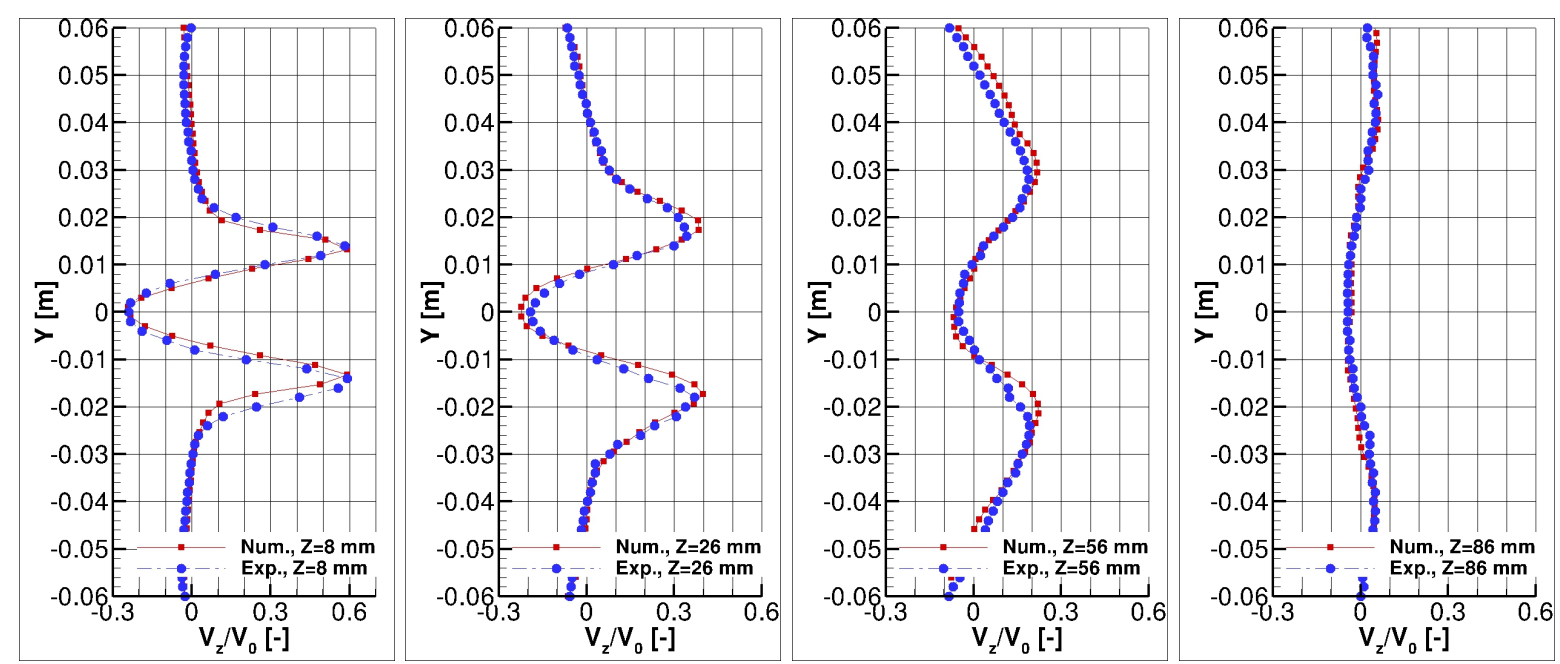

Figure 18. Profiles of mean longitudinal velocity in the channel mid-plane.
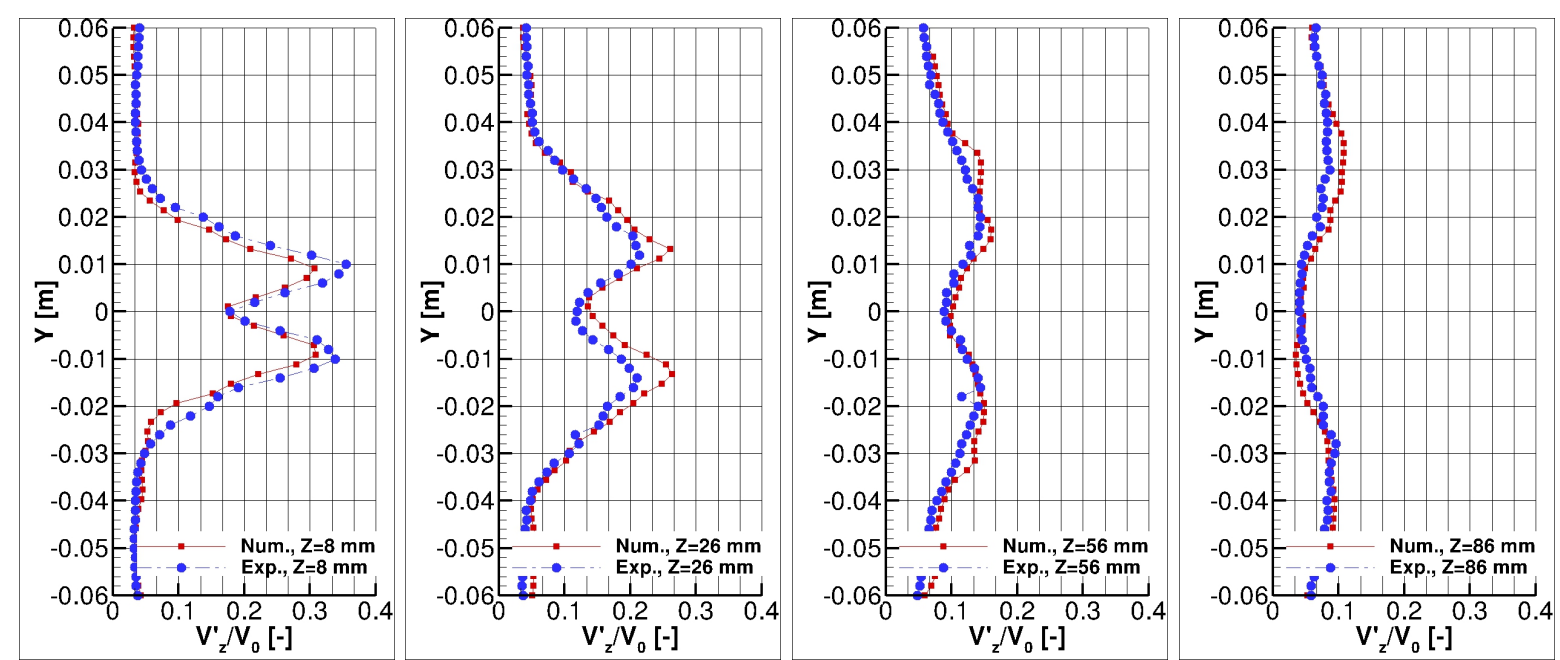

Figure 19. Profiles of longitudinal velocity fluctuations in the channel mid-plane.

Comparisons are quite good for the next two sections. In the last station at $\mathbf{Z}=\mathbf{8 6} \mathbf{~ m m}$, the discrepancies between the measurements and the numerical values of the arithmetic diameter are more important. In this part of the flow, droplets come from, either the upstream injection, or from the lateral walls after an impact. The impact region is located at around 56 millimeters from the chamber end wall, corresponding to the mean position of the lateral recirculation zones closure. In the numerical simulation, the boundary condition does not reproduce the droplet-wall interaction, leading to an underestimation of the largest droplet in the main core of the flow. In any case, the D10 diameter decreases with the distance from the end chamber. Comparisons of the D32 diameter lead to similar conclusions.

The last step consists in the assessment of the IRE experimental data. Since the previous comparisons demonstrate the reliability of the two-phase flow simulation, the assessment is equivalent to comparing the experimental and numerical values of the ethanol gaseous molar concentration. 
The processing of LOS concentrations is similar to the one presented in section 2.5. For each integral concentration distribution from Figure 17, a polynom of degree 3 is calculated as the best fit of measured values. Afterwards, before running the deconvolution algorithm, the polynom is contaminated with random noise with an amplitude equal to the uncertainty value. For each experimental profile, the deconvolution method is run 1000 times in order to calculate the mean profile and the associated standard deviation.

Figure 22 presents the radial profiles of the molar concentration at three sections $Z=26$, 56 and 86 millimeters from the end chamber. For the experimental profiles, the uncertainty values (standard deviation) are shown. A qualitative agreement is obtained in each section between the two approaches with a mean molar concentration varying between 0.4 and $0.6 \mathrm{~mol} / \mathrm{m}^{3}$. The differences between the numerical and experimental values may be as high as $15 \%$ especially at 86 millimeters from the injection plane. The profiles show a slight increase in the concentration from the center to the outer positions. The comparison of experimental profiles clearly shows the increase of the vapor concentration downstream in the flow.
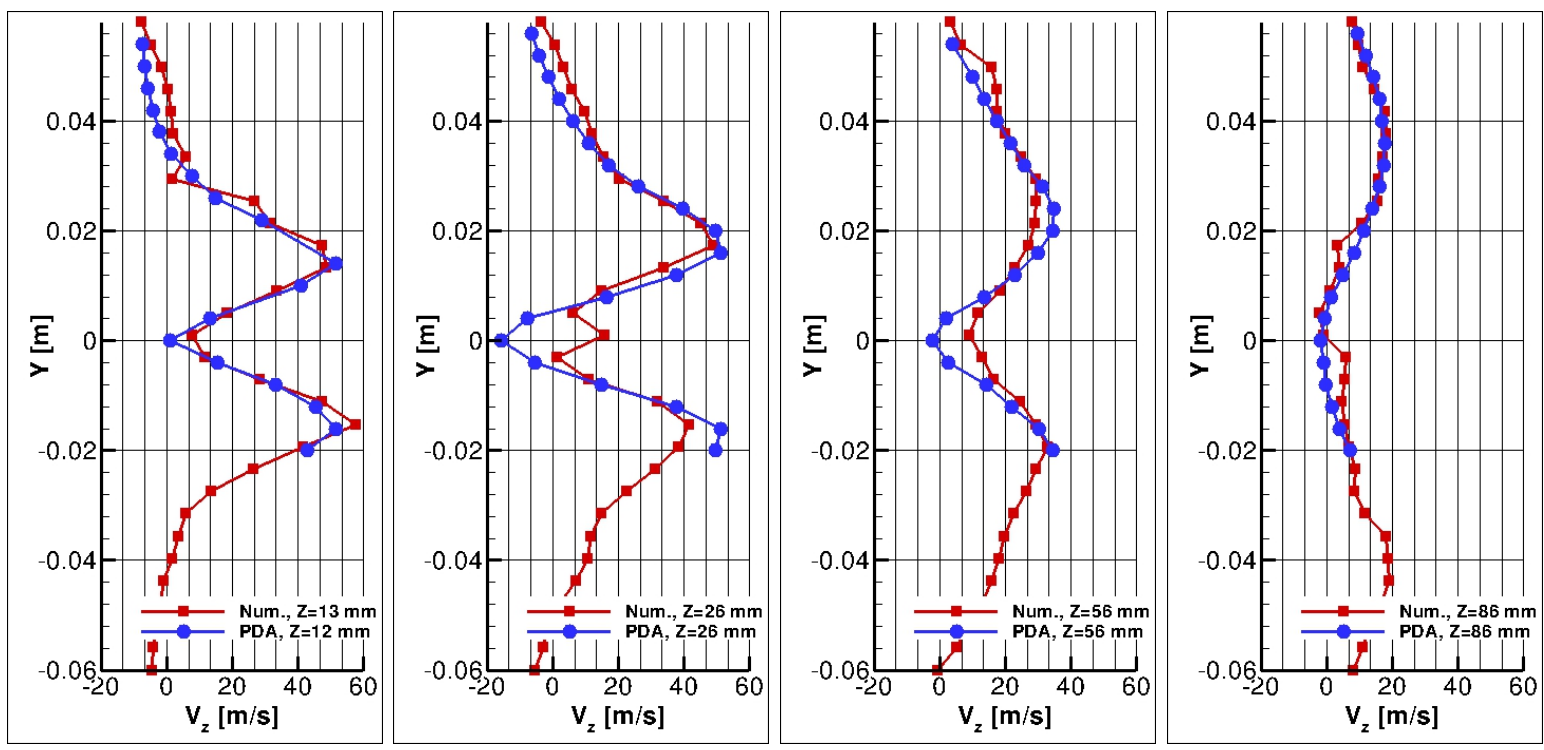

Figure 20. Radial evolution of the droplet mean longitudinal velocity at different distances from the injector nozzle.

\section{Conclusions}

The aim of this paper is to extend the use of IRE to a spray generated behind a real scale injection system. This work is justified by the need to characterize the vaporization of fuels downstream from such an injector, a quantity that is of paramount importance for a combustion chamber. Although some spray characteristics are currently available, like droplet velocities and sizes, thanks to well-known measurement techniques, the vapor concentration is seldom provided due to the presence of liquid droplets. IRE stands out as a quite simple and robust technique to measure the vapor concentration in two-phase flows. Since it is based on the absorption properties of molecules, it is well suited for the investigation of a large number of chemical species, including hydrocarbons. A limitation is caused by the line-of-sight acquisition, which 

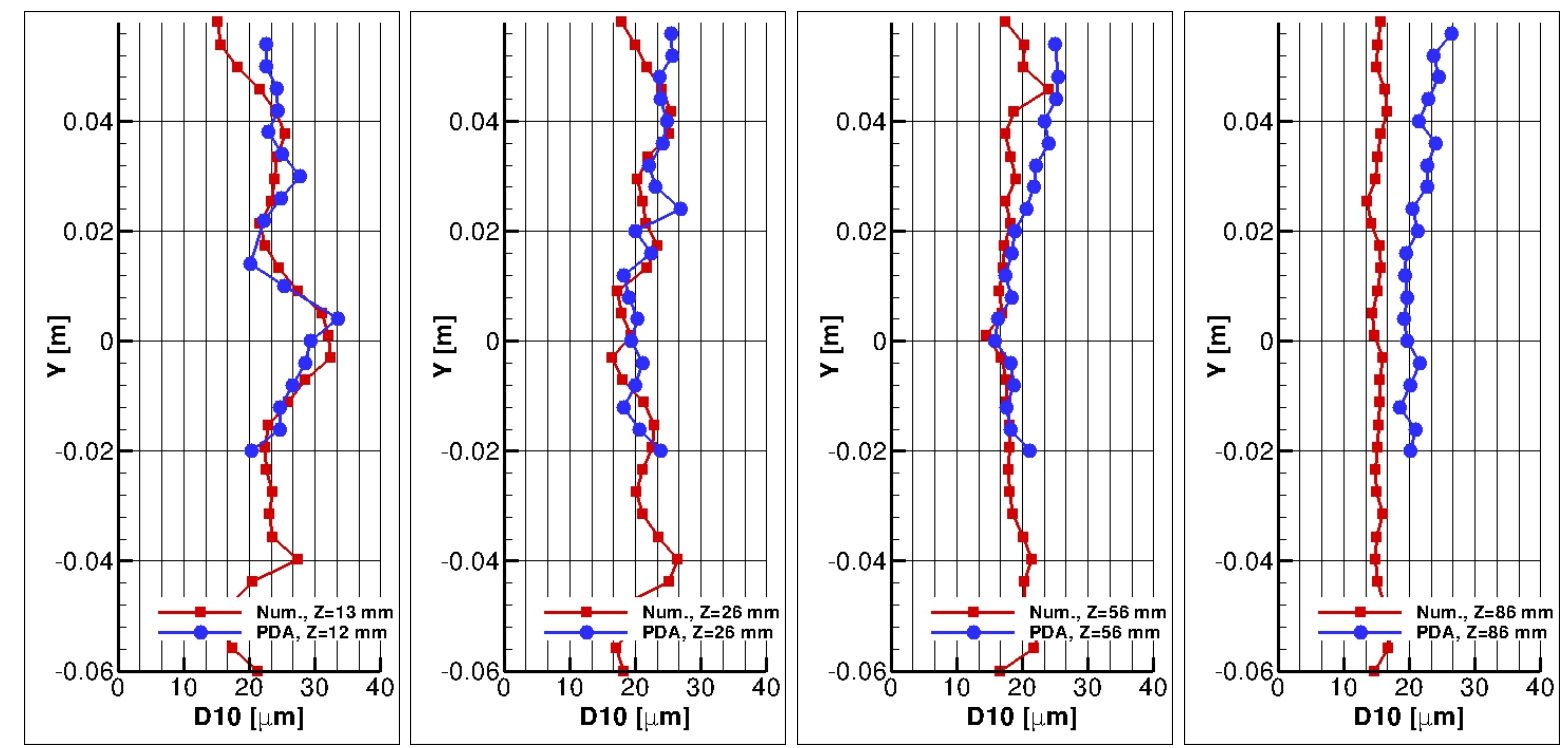

Figure 21. Radial evolution of the droplet mean diameter D10 size at different distances from the injector nozzle.

imposes the complementary use of reconstruction algorithms. Nevertheless, its application to swirled aeronautical injectors is simplified by the axisymmetric geometry of the spray. The assessment of the IRE technique relies on a comparison with a LES numerical simulation. Initially, the numerical results for the gaseous and the dispersed phases were validated by comparison with the measurement data. These comparisons are satisfactory and demonstrate the representativity of the numerical simulation with respect to the two-phase flow. In return, the numerical vapor molar concentration distributions enable the experimental distributions provided by the IRE technique to be assessed. Furthermore, the work proved the capacity of the IRE technique to provide reliable results with uncertainties lower than $15 \%$.

\section{References}

[1] Orain, M., Grisch, F., Jourdanneau, E., Rossow, B., Guin, C. and Tretout, B., C. R. Mécanique, vol 337, pp 373-384, 2009, DOI: $10.1016 /$ j.crm.2009.06.019

[2] Orain, M., Baranger, P., Ledier, C., Apeloig, J. and Grisch, F., Applied Physics B, 2014, DOI: 10.1007/s00340-013-576-z

[3] Adachi, M., McDonell, V.,G. and Samuelsen, G.S., Combustion Science and Technology, vol. 75, pp. 179-194, 1991

[4] Drallmeier, J.,A. and Peters, J.E., Atomization and Sprays, vol 1, pp. 63-88, 1991

[5] Drallmeier, J., Applied Optics, vol. 33, No.30, pp. 7175-7179, 1994

[6] Drallmeier, J.,A. and Billings, T.,P., Atomization and sprays, vol. 4, pp. 99-121, 1994

[7] Wagner, B., - PhD thesis, University of Toulouse, 2009

[8] Fritzer, J., Giuliani, F., Strzelecki, A., Bodoc, V., Journal of Engineering for Gas Turbines and Power J. Eng. Gas Turbines Power, vol. 134, Issue 1, 2012

[9] Lecourt, R., Proceedings of TURBOEXPO 2011, Vancouver, Canada, 2011

[10] Refloch, A. et al., Aerospace Lab Journal, No. 2, 2011, http://www.aerospacelab-journal.org/al2

[11] Skinner, M.,S., SPIE, Vol. 197, 1979 

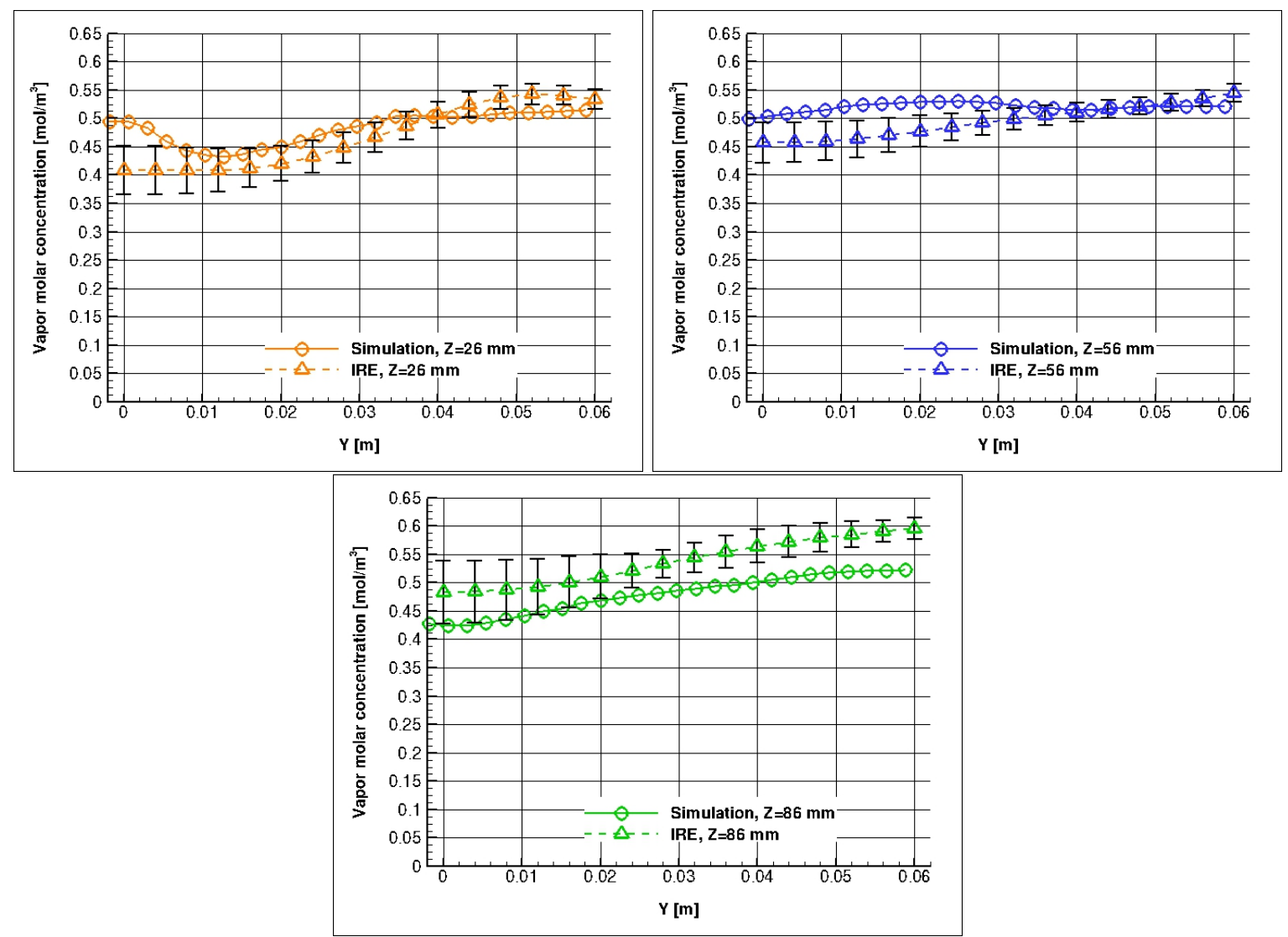

Figure 22. Comparison of ethanol vapor molar concentration profiles at different distances from the injector nozzle.

[12] Chraplyvy, A., R., Applied Optics, vol. 20, No. 15, 1981

[13] Winklhofer, E., and Plimon, A., Optical Engineering, Vol. 30, No. 9, 1991

[14] Billings, T., P. and Drallmeier, J., A., Atomization and Sprays, vol. 4, pp. 99-121, 1994

[15] Golombok, M., et al, Rev.Sci.Instrum., vol. 68, No.11, pp. 4236-4246, 1997.

[16] Zhang, Y., et al., Applied Optics, vol. 39, No.33, pp. 6221-6229, 2000.

[17] Gurton, K., et al., Applied Optics, vol. 44, No.19, pp. 4096-4101, 2005

[18] van de Hulst H. C., Light Scattering by Small Particles, Dover publication, Inc, New York, 1981

[19] Swanson, N., L., and Billard, B., D. and Gennaro, T., L., Applied Optics, vol. 38, No. 27, 1999

[20] Gouesbet, G., et al., Proceedings of an international symposium on optical particle sizing, Rouen, France, 1987

[21] Bodoc, V., and Voicu, D., Proceedings of 17th International Symposium on Applications of Laser Techniques to Fluid Me-chanics, Lisbon, Portugal, 2014

[22] Akesson, E., O. and Daun, K., J., Applied Optics, vol 47, no. 3, 2008

[23] Daun, K., J., Thomson, K., A., Liu, F. and Smallwood, G., J., Applied Optics, vol. 45, No. 19, 2006

[24] Murrone, A. and Villedieu, P., Aerospace Lab Journal, No. 2, 2011, http://www.aerospacelab-journal.org/al2

[25] P. Fede, and O. Simonin, Physics of Fluids, vol. 18, 2006

[26] Lecourt, R., Bismes, F. and Heid, G., ISABE2009, Montreal, Canada, 2009 